\title{
A perturbation result for semi-linear stochastic differential equations in UMD Banach spaces
}

\author{
SONJA GiSEla COX AND ERIKA HAUSENBlAS
}

Abstract. We consider the effect of perturbations of $A$ on the solution to the following semi-linear parabolic stochastic partial differential equation:

$$
\left\{\begin{aligned}
\mathrm{d} U(t) & =A U(t) \mathrm{d} t+F(t, U(t)) \mathrm{d} t+G(t, U(t)) \mathrm{d} W_{H}(t), \quad t>0 \\
U(0) & =x_{0} .
\end{aligned}\right.
$$

Here, $A$ is the generator of an analytic $C_{0}$-semigroup on a UMD Banach space $X, H$ is a Hilbert space, $W_{H}$ is an $H$-cylindrical Brownian motion, $G:[0, T] \times X \rightarrow \mathcal{L}\left(H, X_{\theta_{G}}^{A}\right)$, and $F:[0, T] \times X \rightarrow X_{\theta_{F}}^{A}$ for some $\theta_{G}>-\frac{1}{2}, \theta_{F}>-\frac{3}{2}+\frac{1}{\tau}$, where $\tau \in[1,2]$ denotes the type of the Banach space and $X_{\theta_{F}}^{A}$ denotes the fractional domain space or extrapolation space corresponding to $A$. We assume $F$ and $G$ to satisfy certain global Lipschitz and linear growth conditions.

Let $A_{0}$ denote the perturbed operator and $U_{0}$ the solution to (SDE) with $A$ substituted by $A_{0}$. We provide estimates for $\left\|U-U_{0}\right\|_{L^{p}(\Omega ; C([0, T] ; X))}$ in terms of $D_{\delta}\left(A, A_{0}\right):=\left\|R(\lambda: A)-R\left(\lambda: A_{0}\right)\right\|_{\mathcal{L}\left(X_{\delta-1}^{A}, X\right)}$. Here, $\delta \in[0,1]$ is assumed to satisfy $0 \leq \delta<\min \left\{\frac{3}{2}-\frac{1}{\tau}+\theta_{F}, \frac{1}{2}-\frac{1}{p}+\theta_{G}\right\}$.

The work is inspired by the desire to prove convergence of space approximations of (SDE). In this article, we prove convergence rates for the case that $A$ is approximated by its Yosida approximation.

\section{Introduction}

In this article, we consider the effect of perturbations of $A$ on the solution to the following stochastic partial differential equation:

$$
\left\{\begin{aligned}
\mathrm{d} U(t) & =A U(t) \mathrm{d} t+F(t, U(t)) \mathrm{d} t+G(t, U(t)) \mathrm{d} W_{H}(t), \quad t>0 \\
U(0) & =x_{0} .
\end{aligned}\right.
$$

Here, $A$ is the generator of an analytic $C_{0}$-semigroup $S$ on a UMD Banach space $X, H$ is a Hilbert space, $W_{H}$ is an $H$-cylindrical Brownian motion, $G:[0, T] \times X \rightarrow$ $\mathcal{L}\left(H, X_{\theta_{G}}^{A}\right)$, and $F:[0, T] \times X \rightarrow X_{\theta_{F}}^{A}$ for some $\theta_{G}>-\frac{1}{2}, \theta_{F}>-\frac{3}{2}+\frac{1}{\tau}$, where $\tau \in[1,2]$ denotes the type of the Banach space and $X_{\theta_{F}}^{A}$ denotes the fractional domain space $D\left((-A)^{\theta_{F}}\right)$. We assume $F$ and $G$ to satisfy certain global Lipschitz and linear

Mathematics Subject Classification (2010): 46N40, 35R60, 35A30, 60H15

Keywords: Perturbations, Stochastic differential equations, Stochastic convolutions, Stochastic partial differential equations, Yosida approximation. 
growth conditions, see Sect. 4.1 below. The framework in which we consider (SDE) is precisely the one for which existence and uniqueness of a solution have been proven in the work of VAN NEERVEN, VERAAR AND WEIS [19].

An important example of stochastic partial differential equations that fit into the framework described above are second-order parabolic partial differential equations with multiplicative infinite dimensional noise - in particular, if there is only one spatial dimension, one may consider space-time white noise. For details and more examples, we refer to [19, Section 10].

The main motivation to study the effect of perturbations of $A$ on solutions to equation (SDE) is the desire to prove convergence of certain numerical schemes for approximations in the space dimension. In [4], we demonstrate how the perturbation result proven in this article can be used to obtain pathwise convergence of certain Galerkin and finite element methods for (SDE) in the case that $X$ is Hilbertian. Here, we focus on the theoretical aspects and demonstrate how our perturbation result can be used to prove convergence of the solution processes if $A$ is replaced by its Yosida approximation.

With applications to numerical approximations in mind, we assume the perturbed equation to be set in a (possibly finite dimensional) closed subspace $X_{0}$ of $X$. We assume that there exists a bounded projection $P_{0}: X \rightarrow X_{0}$ such that $P_{0}(X)=X_{0}$. Let $i_{X_{0}}$ be the canonical embedding of $X_{0}$ in $X$, and let $A_{0}$ be a generator of an analytic $C_{0}$-semigroup $S_{0}$ on $X_{0}$. In the setting of numerical approximations, $A_{0}$ would be a suitable restriction of $A$ to the space $X_{0}$.

The perturbed equation we consider is the following stochastic partial differential equation:

$$
\left\{\begin{aligned}
\mathrm{d} U^{(0)}(t)= & A_{0} U^{(0)}(t) \mathrm{d} t+P_{0} F\left(t, U^{(0)}(t)\right) \mathrm{d} t \\
& +P_{0} G\left(t, U^{(0)}(t)\right) \mathrm{d} W_{H}(t), \\
U^{(0)}(0)= & P_{0} x_{0} .
\end{aligned}\right.
$$

Our main result, Theorem 4.3 below, states the following: suppose for some $\delta \geq 0$ satisfying

$$
\delta<\min \left\{\frac{3}{2}-\frac{1}{\tau}+\theta_{F}, \frac{1}{2}+\theta_{G}\right\}
$$

we have, for some $\lambda_{0}$ with $\Re e\left(\lambda_{0}\right)$ sufficiently large, that

$$
D_{\delta}\left(A, A_{0}\right):=\left\|R\left(\lambda_{0}: A\right)-i_{X_{0}} R\left(\lambda_{0}: A_{0}\right) P_{0}\right\|_{\mathcal{L}\left(X_{\delta-1}^{A}, X\right)}<\infty .
$$

Then, provided $x_{0} \in L^{p}\left(\Omega ; \mathcal{F}_{0} ; X_{\delta}^{A}\right)$ for $p \in(2, \infty)$ such that $\frac{1}{p} \leq \frac{1}{2}+\theta_{G}-\delta$, there exists a solution to $\left(\mathrm{SDE}_{0}\right)$ in $L^{p}\left(\Omega ; C\left([0, T] ; X_{0}\right)\right)$, and moreover, there exists a constant $C>0$ such that

$$
\left\|U-i_{X_{0}} U^{(0)}\right\|_{L^{p}(\Omega ; C([0, T] ; X))} \leq C D_{\delta}\left(A, A_{0}\right)\left(1+\left\|x_{0}\right\|_{L^{p}\left(\Omega ; X_{\delta}^{A}\right)}\right) .
$$


As a corollary of Theorem 4.3, we obtain an estimate in the Hölder norm provided we compensate for the initial values (see Corollary 4.6). In short, there exists a constant $C>0$ such that

$$
\begin{aligned}
& \left\|U-S x_{0}-i_{X_{0}}\left(U^{(0)}-S_{0} P_{0} y_{0}\right)\right\|_{L^{p}\left(\Omega ; C^{\lambda}([0, T] ; X)\right)} \\
& \quad \leq C\left\|x_{0}-y_{0}\right\|_{L^{p}(\Omega, X)}+D_{\delta}\left(A, A_{0}\right)\left(1+\left\|x_{0}\right\|_{L^{p}\left(\Omega ; X_{\delta}^{A}\right)}\right),
\end{aligned}
$$

where $0 \leq \lambda<\min \left\{\frac{3}{2}-\frac{1}{\tau}-\left(\delta-\theta_{F}\right)^{+}, \frac{1}{2}-\frac{1}{p}-\left(\delta-\theta_{G}\right)^{+}\right\} ; x^{+}:=\max \{x, 0\}$ for $x \in \mathbb{R}$.

Our results imply that if $\left(A_{n}\right)_{n \in \mathbb{N}}$ is a family of generators of analytic $C_{0}$-semigroups such that the resolvent of $A_{n}$ converges in $\mathcal{L}\left(X_{\delta-1}^{A}, X\right)$ to the resolvent of $A$ for some $\delta \in[0,1]$ (and $\left(A_{n}\right)_{n \in \mathbb{N}}$ is uniformly analytic), then the corresponding solution processes $U_{n}$ converge to the actual solution $U$ in $L^{p}(\Omega ; C([0, T] ; X))$, and the convergence rate is given by $D_{\delta}\left(A, A_{n}\right)$.

In particular, we apply Theorem 4.3 to the Yosida approximation of $A$ which is given by $A_{n}=n A R(n: A)$. In this case, it is necessary to assume that $\theta_{F}$ and $\theta_{G}$ are nonnegative. Let $U^{(n)}$ denote the solution to (SDE) where $A$ is substituted by $A_{n}$. By applying Theorem 4.3 , we obtain that for $\eta>0$ and $p \in(2, \infty)$ such that

$$
\eta<\min \left\{\frac{3}{2}-\frac{1}{\tau}+\theta_{F}, \frac{1}{2}-\frac{1}{p}+\theta_{G}\right\}
$$

we have assuming $x_{0} \in L^{p}\left(\Omega, \mathcal{F}_{0} ; X_{\eta}^{A}\right)$ (see also Theorem 5.1) that there exists a constant $C>0$ such that

$$
\left\|U-U^{(n)}\right\|_{L^{p}(\Omega ; C([0, T] ; X))} \leq C n^{-\min \{\eta, 1\}}\left(1+\left\|x_{0}\right\|_{L^{p}\left(\Omega ; X_{\eta}^{A}\right)}\right) .
$$

To the best of our knowledge, our perturbation results are not yet available even for SDEs in a Hilbert space (i.e., the setting considered in the monograph of DA PRATO AND ZABCZYK [7]). We chose to consider the slightly more complicated UMD space setting because of recent work providing examples of SPDEs for which the UMD space setting seems most suitable, see e.g., aforementioned [19], and the work of SCHNAUBELT AND VERAAR [23].

However, it was proven by KUNZE AND VAN NEERVEN in [14] that if $\left(A_{n}\right)_{n \in \mathbb{N}}$ is a family of generators of analytic $C_{0}$-semigroups such that the resolvent of $A_{n}$ converges to the resolvent of $A$ in the strong operator topology, then the corresponding solution processes $U_{n}$ converge to the actual solution in $L^{p}(\Omega ; C([0, T] ; X))$. The approach taken in that article does not provide convergence rates and requires $\theta_{F}, \theta_{G} \geq 0$.

Another article in which approximations of solutions to (SDE) are considered in the context of perturbations on $A$ is the work of BRZEZNIAK [1]. In that article, it is assumed that $X$ is a UMD space with martingale type 2. In Sect. 5 of that article, the author considers approximations of $A, F, G$, and of the noise. Translated to our setting, the author assumes the perturbed operator $A_{0}$ to satisfy $X_{\theta_{F}}^{A_{0}}=X_{\theta_{F}}^{A}$ and $X_{\theta_{G}}^{A_{0}}=X_{\theta_{G}}^{A}$ (in particular, $X_{0}$ and $X$ must be of the same dimension and if $A_{0}$ is bounded, then $A$ must also be bounded). 
A natural question to ask is how the type of perturbation studied here relates to the perturbations known in the literature. In [8,11], and [22] (see also the monograph by ENGEL AND NAGEL [9, Chapter III.3]), conditions are derived for perturbations of $A$ that lead to an estimate of the type $\left\|S(t)-S_{0}(t)\right\|_{\mathcal{L}(X)}=\mathcal{O}(t)$ as $t \downarrow 0$. These results are comparable to the results we obtain in that Proposition 4.4 below. In particular, [9, Theorem III.3.9] gives precisely the same results as Proposition 4.4, but then for the case that, in the setting of Proposition 4.4, we have $\delta=-1$ and $\theta=0$.

The proof of our perturbation result (Theorem 4.3) requires regularity results for stochastic convolutions. As the convolution under consideration concerns the difference between two semigroups instead of a single semigroup, the celebrated factorization method of DA PRATO, KWAPIEŃ AND ZABCZYK [6] cannot be applied. Therefore, we prove a new result on the regularity of stochastic convolutions, see Lemma 3.2 below. This lemma in combination with some randomized boundedness results on $S-S_{0} P_{0}$ forms the key ingredient of the proof Theorem 4.3.

The setup of this article is as follows: Sect. 2 contains the preliminaries; that is, the relevant results on analytic $C_{0}$-semigroups, vector-valued stochastic integration theory, and $\gamma$-boundedness. In Sect. 3, we present the novel regularity result for stochastic convolutions. We begin Sect. 4 by providing the setting in which our perturbation result is proven, including the precise assumptions on the coefficients in the SDE. We then continue to state and prove our main result, Theorem 4.3. Finally, in Sect. 5, we demonstrate how our main result can be applied by proving convergence for the Yosida approximations.

Notation

Throughout this article $\mathbb{N}:=\{1,2, \ldots\}$. We write $A \lesssim B$ to express that there exists a constant $C>0$ such that $A \leq C B$, and we write $A \approx B$ if $A \lesssim B$ and $B \lesssim A$. For $X$ and $Y$ Banach spaces, we write $X \simeq Y$ if $X$ and $Y$ are isomorphic as Banach spaces.

For $x, y \in \mathbb{R}$, we set $x \vee y:=\max \{x, y\}, x \wedge y:=\min \{x, y\}, x^{+}:=x \vee 0$, and, for $x \geq 0,\lceil x\rceil:=\inf \{n \in \mathbb{N} \cup\{0\}: n \geq x\}$.

Let $Y$ be a Banach space. For $T>0$ and $\beta \in(0,1)$, we take the following definition for the Hölder norm of $Y$-valued functions:

$$
\|f\|_{C^{\beta}([0, T] ; Y)}:=\|f(0)\|_{Y}+\sup _{0 \leq s<t \leq T} \frac{\|f(t)-f(s)\|_{Y}}{(t-s)^{\beta}} .
$$

For the definition of the space $V_{\mathrm{c}}^{\alpha, p}([a, b] \times \Omega ; Y)$, we refer to Definition 2.6.

For $X, Y$ Banach spaces, we let $\mathcal{L}(X, Y)$ be the Banach space of all bounded linear operators from $X$ to $Y$ endowed with the operator norm. For brevity, we set $\mathcal{L}(X):=$ $\mathcal{L}(X, X)$. For $A: D(A) \subset X \rightarrow X$ a linear operator on $X$, we denote the resolvent set of $A$ by $\varrho(A)$, i.e., $\varrho(A) \subset \mathbb{C}$ is the set of all the complex numbers $\lambda \in \mathbb{C}$ for which $\lambda I-A$ is boundedly invertible on (the complexification of) $X$. For $\lambda \in \varrho(A)$, we denote the resolvent of $A$ in $\lambda$ by $R(\lambda: A)$, i.e., $R(\lambda: A)=(\lambda I-A)^{-1}$. The spectrum of $A$, i.e., the complement of $\varrho(A)$ in $\mathbb{C}$, is denoted by $\sigma(A)$. Finally, $X_{\theta}^{A}$ 
denotes the fractional domain space of the operator $A$ for $\theta>0$, and the fractional extrapolation space for $\theta<0$. See also Sect. 2.1.

\section{Preliminaries}

Throughout this section, $X$ denotes a Banach spaces and $H$ denotes a Hilbert space.

\subsection{Analytic semigroups}

For $\delta \in[0, \pi]$, we define

$$
\Sigma_{\delta}:=\{z \in \mathbb{C} \backslash\{0\}:|\arg (z)|<\delta\}
$$

DEFINITION 2.1. Let $X$ be a Banach space, let $\delta \in(0, \pi]$, and let $(S(t))_{t \in[0, \infty)} \subset$ $\mathcal{L}(X)$ be a $C_{0}$-semigroup on $X$. We say that $S$ is analytic on $\Sigma_{\delta}$ if $t \mapsto S(t)$ extends to $\Sigma_{\delta}$ analytically and for all $x \in X$ one has

$$
\lim _{z \in \Sigma_{\delta}, z \rightarrow 0} S(z) x=x
$$

We say that $S$ is an analytic $C_{0}$-semigroup if there exists a $\delta \in(0, \pi]$ such that $S$ is analytic on $\Sigma_{\delta}$.

It is not difficult to check that if the $C_{0}$-semigroup $S$ is analytic on a sector $\Sigma_{\delta}$, then it satisfies the semigroup property on that sector. The theorem below is obtained from [20, Theorem 2.5.2] by straightforward adaptations and gives some characterizations of analytic $C_{0}$-semigroups that we need.

THEOREM 2.2. Let $A$ be the generator of a $C_{0}$-semigroup $(S(t))_{t \geq 0}$ on $X$. Let $\omega \in \mathbb{R}$ be such that $\left(e^{-\omega t} S(t)\right)_{t \geq 0}$ is bounded. The following statements are equivalent:

(i) $S$ is an analytic $C_{0}$-semigroup on $\Sigma_{\delta}$ for some $\delta \in\left(0, \frac{\pi}{2}\right]$.

(ii) There exists a $\theta \in\left(0, \frac{\pi}{2}\right]$ such that $\omega+\Sigma_{\frac{\pi}{2}+\theta} \subset \varrho(A)$, and there exists a constant $K_{\theta}>0$ such that

$$
|\lambda-\omega|\|R(\lambda: A)\|_{\mathcal{L}(X)} \leq K_{\theta}, \quad \text { for all } \lambda \in \omega+\Sigma_{\frac{\pi}{2}+\theta}
$$

(iii) $S$ is differentiable for $t>0$ (in the uniform operator topology), $S^{\prime}=A S$, and there exists a constant $C$ such that

$$
t\|A S(t)\|_{\mathcal{L}(X)} \leq C e^{\omega t}, \quad \text { for all } t>0
$$

Moreover, we have $\delta_{\text {sup }}=\theta_{\text {sup }}$, where $\delta_{\text {sup }}$ is the supremum over all $\delta$ such that (i) holds and $\theta_{\text {sup }}$ the supremum over all $\theta$ such that (ii) holds.

The theorem above justifies the following definition: 
DEFINITION 2.3. Let $A$ be the generator of an analytic $C_{0}$-semigroup on $X$. We say that $A$ is of type $(\omega, \theta, K)$, where $\omega \in \mathbb{R}, \theta \in\left(0, \frac{\pi}{2}\right]$ and $K>0$, if $\omega+\Sigma_{\frac{\pi}{2}+\theta} \subseteq$ $\varrho(A),\left(e^{\omega t} S(t)\right)_{t \geq 0}$ is bounded, and

$$
|\lambda-\omega|\|R(\lambda: A)\|_{\mathcal{L}(X)} \leq K \text { for all } \lambda \in \omega+\Sigma_{\frac{\pi}{2}+\theta} .
$$

$R E M A R K 2.4$. Let $A$ be of type $(\omega, \theta, K)$ for some $\omega \in \mathbb{R}, \theta \in\left(0, \frac{\pi}{2}\right]$ and $K>0$. It follows from the aforementioned proof in [20] that one may take $C=\frac{K}{\pi \cos \theta}$ in part (iii) of Theorem 2.2, and that for every $\theta^{\prime} \in[0, \theta)$, there exists a constant $C_{\theta^{\prime}}$ depending only on $\theta$ and $K$ such that

$$
\left\|e^{-\omega z} S(z)\right\|_{\mathcal{L}(X)} \leq C_{\theta^{\prime}}, \quad \text { for all } z \in \Sigma_{\theta^{\prime}} .
$$

If $A$ is the generator of an analytic $C_{0}$-semigroup of type $(\omega, \theta, K)$ and

$$
\lambda \in 2|\omega|(\cos \theta)^{-1}+\Sigma_{\frac{\pi}{2}+\theta},
$$

then $|\lambda|>2|\omega|$ and hence, $|\lambda-\omega|>|| \lambda|-| \omega|| \geq \frac{1}{2}|\lambda|$, whence

$$
\|A R(\lambda: A)\|_{\mathcal{L}(X)}=\|\lambda R(\lambda: A)-I\|_{\mathcal{L}(X)} \leq 1+2 K .
$$

Let $A$ be a generator of an analytic $C_{0}$-semigroup of type $(\omega, \theta, K)$ on $X$. We define the extrapolation spaces of $A$ as in [20, Section 2.6]; that is, for $\delta>0$ and $\lambda \in \mathbb{C}$ such that $\Re e(\lambda)>\omega$ we define $X_{-\delta}^{A}$ to be the completion of $X$ under the norm

$$
\|x\|_{X_{-\delta}^{A}}:=\left\|(\lambda I-A)^{-\delta} x\right\|_{X} .
$$

We also define the fractional domain spaces of $A$, i.e., for $\delta>0$ we define $X_{\delta}^{A}=$ $D\left((\lambda I-A)^{\delta}\right)$ and

$$
\|x\|_{X_{\delta}^{A}}:=\left\|(\lambda I-A)^{\delta} x\right\|_{X} .
$$

One may check that regardless of the choice of $\lambda$ the extrapolation spaces and the fractional domain spaces are uniquely determined up to isomorphisms: for $\delta>0$ and $\lambda, \mu \in \mathbb{C}$ such that $\Re e(\lambda), \Re e(\mu)>\omega$ one has $(\lambda I-A)^{\delta}(\mu I-A)^{-\delta} \in \mathcal{L}(X)$ and

$$
\left\|(\lambda I-A)^{\delta}(\mu I-A)^{-\delta}\right\|_{\mathcal{L}(X)} \leq C(\omega, \theta, K, \lambda, \mu),
$$

where $C(\omega, \theta, K, \lambda, \mu)$ denotes a constant depending only on $\omega, \theta, K, \lambda$, and $\mu$. Moreover, for $\delta, \beta \in \mathbb{R}$ one has $(\lambda I-A)^{\delta}(\lambda I-A)^{\beta}=(\lambda I-A)^{\delta+\beta}$ on $X_{\gamma}^{A}$, where $\gamma=\max \{\beta, \delta+\beta\}$ (see [20, Theorem 2.6.8]).

Statement (iii) in Theorem 2.2 can be extended; from the proof of [20, Theorem 2.6.13], we obtain that for an analytic $C_{0}$-semigroup $S$ of type $(\omega, \theta, K)$ generated by $A$ one has, for $\delta>0$ and $\omega^{\prime}>\omega$, that

$$
\left\|\left(\omega^{\prime} I-A\right)^{\delta} S(t)\right\|_{\mathcal{L}\left(X, X_{\delta}^{A}\right)} \leq 2\left(\frac{K}{\pi \cos \theta}\right)^{\lceil\delta\rceil} t^{-\delta} e^{\omega t}
$$

for all $t>0$. Finally, [20, Theorem 2.6.10] states the following interpolation result for $x \in D(A)$ and $\delta \in(0,1)$ :

$$
\left\|(\lambda I-A)^{\delta} x\right\|_{\mathcal{L}(X)} \leq 2(1+K)\|x\|_{X}^{1-\delta}\|(\lambda I-A) x\|_{X}^{\delta} .
$$




\subsection{Stochastic integration in Banach spaces}

Let $\left(\Omega,\left(\mathcal{F}_{t}\right)_{t \geq 0}, \mathbb{P}\right)$ denote a probability space endowed with a filtration $\left(\mathcal{F}_{t}\right)_{t \geq 0}$. We recall the basics concerning stochastic integration in UMD Banach spaces as presented in the work of VAN NEERVEN, VERAAR AND WEIS [18].

Recall that the UMD property is a geometric Banach space property that is satisfied by all Hilbert spaces and by the 'classical' reflexive function spaces, e.g., the $L^{p}$-spaces and Sobolev spaces $W^{k, p}$ for $k \in \mathbb{N}$ and $p \in(1, \infty)$. For the precise definition of the UMD property and for a more elaborate treatment of spaces satisfying this property, we refer to [2]. For this article, it is relevant that the UMD property is maintained under Banach space isomorphisms.

Fix $T>0$. An $H$-cylindrical Brownian motion over $\left(\Omega,\left(\mathcal{F}_{t}\right)_{t \geq 0}, \mathbb{P}\right)$ is a linear mapping $W_{H}: L^{2}(0, T ; H) \rightarrow L^{2}(\Omega)$ with the following properties:

(i) for all $h \in L^{2}(0, T ; H)$ the random variable $W_{H}(h)$ is Gaussian;

(ii) for all $h_{1}, h_{2} \in L^{2}(0, T ; H)$ we have $\mathbb{E} W_{H}\left(h_{1}\right) W_{H}\left(h_{2}\right)=\left\langle h_{1}, h_{2}\right\rangle$;

(iii) for all $h \in H$ and all $t \in[0, T]$ we have that $W_{H}\left(1_{[0, t]} \otimes h\right)$ is $\mathcal{F}_{t}$-measurable;

(iv) for all $h \in H$ and all $s, t \in[0, T], s \leq t$ we have that $W_{H}\left(1_{[s, t]} \otimes h\right)$ is independent of $\mathcal{F}_{S}$.

Formally, an $H$-cylindrical Brownian motion can be thought of as a standard Brownian motion in the Hilbert space $H$.

Let $0 \leq t_{1} \leq t_{2} \leq T, B \in \mathcal{F}_{t_{1}}, x \in X$, and $h \in H$. By $x \otimes h$, we denote the element of $\mathcal{L}(H, X)$ defined by $(x \otimes h) g=\langle h, g\rangle x, g \in H$. For a process $\Phi:[0, T] \times \Omega \rightarrow \mathcal{L}(H, X)$ of the form

$$
\Phi(t, \omega):=1_{B}(\omega) 1_{\left[t_{1}, t_{2}\right)}(t)(x \otimes h)
$$

we define the stochastic integral of $\Phi$ against $W_{H}$ by

$$
\int_{0}^{T} \Phi \mathrm{d} W_{H}:=1_{B} W_{H}\left(1_{\left[t_{1}, t_{2}\right)} \otimes h\right) x .
$$

By linearity, we can extend the definition of a stochastic integral to any process constructed by a finite sum of elements of the type (5). We refer to such a process as an adapted elementary process.

Let $\mathcal{H}$ be a Hilbert space (we take $\mathcal{H}=L^{2}(0, T ; H)$ below). The Banach space $\gamma(\mathcal{H}, X)$ is defined as the completion of $\mathcal{H} \otimes X$ with respect to the norm

$$
\left\|\sum_{n=1}^{N} h_{n} \otimes x_{n}\right\|_{\gamma(\mathcal{H}, X)}^{2}:=\mathbb{E}\left\|\sum_{n=1}^{N} \gamma_{n} x_{n}\right\|_{X}^{2} .
$$

Here, we assume that $\left(h_{n}\right)_{n=1}^{N}$ is an orthonormal sequence in $\mathcal{H},\left(x_{n}\right)_{n=1}^{N}$ is a sequence in $X$, and $\left(\gamma_{n}\right)_{n=1}^{N}$ is a standard Gaussian sequence on some probability space. The space $\gamma(H, X)$ embeds continuously into $\mathcal{L}(\mathcal{H}, X)$, and its elements are referred to 
as the $\gamma$-radonifying operators from $\mathcal{H}$ to $X$. For properties of this norm and further details, we refer to the survey paper by VAN NEERVEN [16].

Let $-\infty<a<b<\infty$, then $\gamma(a, b ; H, X)$ and $\gamma(a, b ; X)$ are used as short-hand notation for $\gamma\left(L^{2}(a, b ; H), X\right)$ and $\gamma\left(L^{2}(a, b), X\right)$, respectively. More generally, for $(R, \mathcal{R}, \mu)$ a $\sigma$-finite measure space the notation $\gamma(R ; H, X)$ and $\gamma(R ; X)$ is used for $\gamma\left(L^{2}(R ; H), X\right)$ and $\gamma\left(L^{2}(R), X\right)$. If $X$ is a Hilbert space, and $(R, \mathcal{R}, \mu)$ is a $\sigma$-finite measure space, then $\gamma(R ; H, X) \simeq L^{2}\left(R ; \mathcal{L}_{2}(H, X)\right)$ where $\mathcal{L}_{2}(H, X)$ denotes the space of Hilbert-Schmidt operators from $H$ to $X$.

A process $\Phi:[0, \infty) \times \Omega \rightarrow \mathcal{L}(H, X)$ is called $H$-strongly measurable if for every $h \in H$ the process $\Phi h$ is strongly measurable. The process is called adapted if $\Phi h$ is adapted for each $h \in H$. Concerning stochastic integrability of such processes, we cite [18, Theorem 3.6]:

THEOREM 2.5. ( $L^{p}$-stochastic integrability) Let $p \in(1, \infty)$ and $T>0$ be fixed. For an $H$-strongly measurable adapted process $\Phi:(0, T) \times \Omega \rightarrow \mathcal{L}(H, X)$ such that $\Phi^{*} x^{*} \in L^{p}\left(\Omega ; L^{2}(0, T ; H)\right)$ for all $x^{*} \in X^{*}$ the following are equivalent:

(i) There exists a sequence of elementary adapted processes $\left(\Phi_{n}\right)_{n \in \mathbb{N}}$ such that $x^{*}\left(\Phi_{n} h\right) \rightarrow x^{*}(\Phi h)$ in measure on $(0, T) \times \Omega$ as $n \rightarrow \infty$, and there exists a random variable $\eta \in L^{p}(\Omega, X)$ such that

$$
\eta=\lim _{n \rightarrow \infty} \int_{0}^{T} \Phi_{n} \mathrm{~d} W_{H} \quad \text { in } L^{p}(\Omega, X) ;
$$

(ii) there exists a (necessarily unique) $R_{\Phi} \in L^{p}(\Omega ; \gamma(0, T ; H, X))$ such that for all $x^{*} \in X^{*}$ we have $R_{\Phi}^{*} x^{*}=\Phi^{*} x^{*}$ in $L^{p}\left(\Omega ; L^{2}(0, T ; H)\right)$.

In the situation that the equivalent statements above hold we say that $\Phi$ is $L^{p}$-stochastically integrable and we set $\int_{0}^{T} \Phi \mathrm{d} W_{H}(t):=\eta$.

If $\Phi:(0, T) \times \Omega \rightarrow \mathcal{L}(H, X)$ is $L^{p}$-stochastically integrable then the following estimate holds:

$$
\mathbb{E} \sup _{0 \leq t \leq T}\left\|\int_{0}^{t} \Phi \mathrm{d} W_{H}\right\|_{X}^{p} \bar{\sim}_{p, X} \mathbb{E}\left\|R_{\Phi}\right\|_{\gamma(0, T ; H, X)}^{p},
$$

the implied constants depending only on the Banach space $X$ and on $p$.

From now on, if $\Phi$ is $L^{p}$-stochastically integrable for some $p \in(1, \infty)$, we simply use $\Phi$ to denote both a process and the (unique) $R_{\Phi} \in L^{p}(\Omega ; \gamma(0, T ; H, X))$ that satisfies $R_{\Phi}^{*} x^{*}=\Phi^{*} x^{*}$ in $L^{p}\left(\Omega ; L^{2}(0, T ; H)\right)$ for all $x^{*} \in X^{*}$.

In [19], existence and uniqueness of a solution to a semi-linear stochastic partial differential equation are given in a space of continuous, 'weighted' $L^{p}$-stochastically integrable processes. The precise definition of this space, which is also used in this article, is provided below. 
DEFINITION 2.6. For $\alpha \in\left[0, \frac{1}{2}\right), 1 \leq p<\infty$ and $0 \leq a \leq b<\infty$, we denote by $V_{\mathrm{c}}^{\alpha, p}([a, b] \times \Omega ; X)$ the space of adapted, continuous processes $\Phi:[a, b] \times \Omega \rightarrow X$ for which the following norm is finite:

$\|\Phi\|_{V_{\mathrm{c}}^{\alpha, p}([a, b] \times \Omega ; X)}=\|\Phi\|_{L^{p}(\Omega ; C([a, b] ; X))}+\sup _{a \leq t \leq b}\left\|s \mapsto(t-s)^{-\alpha} \Phi(s)\right\|_{L^{p}(\Omega ; \gamma(a, t ; X))}$.

One easily checks that for $0 \leq \beta \leq \alpha<\frac{1}{2}$ and $\Phi \in V_{\mathrm{c}}^{\alpha, p}([a, b] \times \Omega ; X)$ one has

$$
\|\Phi\|_{V_{\mathrm{c}}^{\beta, p}([a, b] \times \Omega ; X)} \leq(b-a)^{\alpha-\beta}\|\Phi\|_{V_{\mathrm{c}}^{\alpha, p}([a, b] \times \Omega ; X)} .
$$

Note also that we have $V_{\mathrm{c}}^{\alpha, p}([0, T] \times \Omega ; X) \subset L^{p}(\Omega ; C([0, T] ; X))$. On the other hand, the lemma below provides an embedding in the opposite direction. This embedding depends on the type $\tau \in[1,2]$ of the Banach space $X$. We refer to [15] for a precise definition of type (and co-type) and further details. In what follows, it is relevant that every Banach space has type $\tau$ for some $\tau \in[1,2]$, and that if a Banach space has type $\tau_{0} \in[1,2]$, then it has type $\tau$ for all $\tau \in\left[0, \tau_{0}\right]$. Moreover, we use that the type of a Banach space is preserved under Banach space isomorphisms.

For a proof of the following lemma, see [19, Lemma 3.3].

LEMMA 2.7. Let $X$ be a Banach space with type $\tau$. Then for all $T>0, \varepsilon>0$ and $\alpha \in\left[0, \frac{1}{2}\right)$ one has

$$
L^{p}\left(\Omega ; C^{\frac{1}{\tau}-\frac{1}{2}+\varepsilon}([0, T] ; X)\right) \hookrightarrow V_{\mathrm{c}}^{\alpha, p}([0, T] \times \Omega ; X) .
$$

\section{3. $\gamma$-Boundedness}

For vector-valued stochastic integrals, the concept of $\gamma$-boundedness plays the role that uniform boundedness does for ordinary integrals: the Kalton-Weis multiplier theorem (Proposition 2.10 below) allows one to estimate terms out of a stochastic integral, provided they are $\gamma$-bounded.

Throughout this section, let $X$ and $Y$ denote Banach spaces. A family $\mathscr{B} \subset \mathcal{L}(X, Y)$ is called $\gamma$-bounded if there exists a constant $C$ such that for all $N \geq 1$, all $x_{1}, \ldots, x_{N} \in$ $X$, and all $B_{1}, \ldots, B_{N} \in \mathscr{B}$ we have

$$
\mathbb{E}\left\|\sum_{n=1}^{N} \gamma_{n} B_{n} x_{n}\right\|_{Y}^{2} \leq C^{2} \mathbb{E}\left\|\sum_{n=1}^{N} \gamma_{n} x_{n}\right\|_{X}^{2} .
$$

The least admissible constant $C$ is called the $\gamma$-bound of $\mathscr{B}$, notation: $\gamma_{[X, Y]}(\mathscr{B})$. Note that any $\gamma$-bounded set of operators is automatically uniformly bounded, and the reverse holds if $X$ is a Hilbert space.

The following lemma is a direct consequence of the Kahane contraction principle:

LEMMA 2.8. If $\mathscr{B} \subset \mathcal{L}(X, Y)$ is $\gamma$-bounded and $M>0$ then $M \mathscr{B}:=\{a B: a \in$ $[-M, M], B \in \mathscr{B}\}$ is $\gamma$-bounded with $\gamma_{[X, Y]}(M \mathscr{B}) \leq M \gamma_{[X, Y]}(\mathscr{B})$. 
The following proposition, which is a variation of a result of WEIS [24, Proposition 2.5], gives a sufficient condition for $\gamma$-boundedness.

PROPOSITION 2.9. Let $f:[0, T] \rightarrow \mathcal{L}(X, Y)$ be a function such that for all $x \in X$ the function $t \mapsto f(t) x$ is continuously differentiable on $(0, T)$. Suppose $g \in L^{1}(0, T)$ is such that for all $t \in(0, T)$

$$
\left\|f^{\prime}(t) x\right\|_{Y} \leq g(t)\|x\|_{X}, \quad \text { for all } x \in X
$$

Then the set $\mathscr{R}:=\{f(t): t \in(0, T)\}$ is $\gamma$-bounded in $\mathscr{L}(X, Y)$ and

$$
\gamma_{[X, Y]}(\mathscr{R}) \leq\|f(0)\|_{\mathcal{L}(X, Y)}+\|g\|_{L^{1}(0, T)} .
$$

The following $\gamma$-multiplier result, due to KALTON AND WEIS [12] (see also [16]), establishes a relation between stochastic integrability and $\gamma$-boundedness.

PROPOSITION 2.10. ( $\gamma$-Multiplier theorem) Suppose $X$ does not contain a closed subspace isomorphic to $c_{0}$. Suppose $M:(0, T) \rightarrow \mathcal{L}(X, Y)$ is an $X$ strongly measurable function with $\gamma$-bounded range $\mathcal{M}=\{M(t): t \in(0, T)\}$. If $\Phi \in \gamma(0, T ; H, X)$ then $M \Phi \in \gamma(0, T ; H, Y)$ and

$$
\|M \Phi\|_{\gamma(0, T ; H, Y)} \leq \gamma_{[X, Y]}(\mathcal{M})\|\Phi\|_{\gamma(0, T ; H, X)} .
$$

In all applications in this paper, $X$ is a UMD space and therefore does not contain a copy of $c_{0}$. We refer to [16] for details.

Finally, we recall the following $\gamma$-boundedness estimate for analytic $C_{0}$-semigroups (see e.g., [19, Lemma 4.1]).

LEMMA 2.11. Let $X$ be a Banach space and let $A$ be the generator of an analytic $C_{0}$-semigroup $S$ of type $(\omega, \theta, K)$ on $X$. Then for all $0 \leq \beta<\alpha$ and $T>0$ there exists a constant $C$ depending on $S$ only in terms of $\omega, \theta$, and $K$, such that for all $t \in(0, T]$ the set $\mathscr{S}_{\alpha, t}=\left\{s^{\alpha} S(s): s \in[0, t]\right\}$ is $\gamma$-bounded in $\mathcal{L}\left(X, X_{\beta}^{A}\right)$ and we have

$$
\gamma_{\left[X, X_{\beta}^{A}\right]}\left(\mathscr{S}_{\alpha, t}\right) \leq C t^{\alpha-\beta}, \quad t \in(0, T]
$$

Note that the constant $C$ in the lemma above may depend on $T$.

\section{Estimates for (stochastic) convolutions}

In this section, we provide the estimates for (stochastic) convolutions needed to derive the perturbation result given in Theorem 4.3. In order to avoid confusion further on, we use $Y_{1}$ and $Y_{2}$ to denote UMD Banach spaces in this section. Moreover, we adopt the notation introduced in Sect. 2.2; that is, $H$ denotes a Hilbert space, and $W_{H}$ denotes a $H$-cylindrical Brownian motion over a probability space $\left(\Omega,\left(\mathcal{F}_{t}\right)_{t \geq 0}, \mathbb{P}\right)$.

The following lemma is proven in [5]. It is an adaptation of [19, Proposition 4.5]. 
LEMMA 3.1. Let $(R, \mathcal{R}, \mu)$ be a finite measure space and $(S, \mathcal{S}, v)$ a $\sigma$-finite measure space. Let $\Phi_{1}:[0, T] \times \Omega \rightarrow \mathcal{L}\left(H, Y_{1}\right)$, let $\Phi_{2} \in L^{1}\left(R ; \mathcal{L}\left(Y_{1}, Y_{2}\right)\right)$, and let $f \in L^{\infty}\left(R \times[0, T] ; L^{2}(S)\right)$. If $\Phi_{1}$ is $L^{p}$-stochastically integrable for some $p \in(1, \infty)$, then

$$
\begin{aligned}
& \left\|s \mapsto \int_{0}^{T} \int_{R} f(r, u)(s) \Phi_{2}(r) \Phi_{1}(u) d \mu(r) \mathrm{d} W_{H}(u)\right\|_{L^{p}\left(\Omega ; \gamma\left(S ; Y_{2}\right)\right)} \\
& \lesssim \operatorname{ess} \sup _{(r, u) \in R \times[0, T]}\|f(r, u)\|_{L^{2}(S)}\left\|\Phi_{2}\right\|_{L^{1}\left(R, \mathcal{L}\left(Y_{1}, Y_{2}\right)\right)}\left\|\Phi_{1}\right\|_{L^{p}\left(\Omega ; \gamma\left(0, T ; H, Y_{1}\right)\right)},
\end{aligned}
$$

with implied constant depending only on $p, Y_{1}$, and $Y_{2}$, provided the right-hand side is finite.

To our knowledge, most regularity results for stochastic convolutions are based on the factorization method introduced in [6]. The result below is based on the regularity of the convolving functions.

LEMMA 3.2. Let $T>0, p \in(1, \infty)$ and $\eta \in(0,1)$. Suppose the process $\Phi \in$ $L^{p}\left(\Omega ; \gamma\left(0, T ; H, Y_{1}\right)\right)$ is adapted to $\left(\mathcal{F}_{t}\right)_{t \geq 0}$ and satisfies

$$
\sup _{0 \leq t \leq T}\left\|s \mapsto(t-s)^{-\eta} \Phi(s)\right\|_{L^{p}\left(\Omega ; \gamma\left(0, t ; H, Y_{1}\right)\right)}<\infty .
$$

Let $\Psi:[0, T] \rightarrow \mathcal{L}\left(Y_{1}, Y_{2}\right)$ be such that $\Psi x$ is continuously differentiable on $(0, T)$ for all $x \in Y_{1}$. Suppose moreover that there exists a function $g \in L^{1}(0, T)$ and a constant $0<\theta<\eta$ such that for all $v \in(0, T)$ we have

$$
v^{\theta}\left\|\Psi^{\prime}(v) x\right\|_{Y_{2}}+\theta v^{\theta-1}\|\Psi(v) x\|_{Y_{2}} \leq g(v)\|x\|_{Y_{1}}, \text { for all } x \in Y_{1} .
$$

Then the stochastic convolution process $t \mapsto \int_{0}^{t} \Psi(t-s) \Phi(s) \mathrm{d} W_{H}(s)$ is well-defined and

$$
\begin{aligned}
\| t & \mapsto \int_{0}^{t} \Psi(t-s) \Phi(s) \mathrm{d} W_{H}(s) \|_{C^{\eta-\theta}\left([0, T] ; L^{p}\left(\Omega ; Y_{2}\right)\right)} \\
& \leq 2 \bar{C}_{p}\|g\|_{L^{1}(0, T)} \sup _{0 \leq t \leq T}\left\|s \mapsto(t-s)^{-\eta} \Phi(s)\right\|_{L^{p}\left(\Omega ; \gamma\left(0, t ; H, Y_{1}\right)\right)},
\end{aligned}
$$

where $\bar{C}_{p}$ is the constant in the upper estimate of equation (6) for the pth moment, for the space $Y_{1}$.

$R E M A R K$ 3.3. By a straightforward adaptation of the proof of Lemma 3.2 provided below, one may check that if one takes $\theta=0$ in the setting of Lemma 3.2, it holds that

$$
\begin{aligned}
\| t & \mapsto \int_{0}^{t} \Psi(t-s) \Phi(s) \mathrm{d} W_{H}(s) \|_{C^{\eta}\left([0, T] ; L^{p}\left(\Omega ; Y_{2}\right)\right)} \\
& \leq\left(2 \bar{C}_{p}\|g\|_{L^{1}(0, T)}+\|\Psi(0)\|_{\left.L\left(Y_{1}, Y_{2}\right)\right)}\right) \sup _{0 \leq t \leq T}\left\|s \mapsto(t-s)^{-\eta} \Phi(s)\right\|_{L^{p}\left(\Omega ; \gamma\left(0, t ; H, Y_{1}\right)\right)} .
\end{aligned}
$$

Moreover, in the setting of Lemma 3.2, one may also take $g \in L^{q^{\prime}}(0, T)$ and $\Phi$ such that 


$$
\int_{0}^{T}\left\|s \mapsto(t-s)^{-\eta} \Phi(s)\right\|_{L^{p}\left(\Omega ; \gamma\left(0, t ; H, Y_{1}\right)\right)}^{q} \mathrm{~d} t<\infty,
$$

where $q \in[1, \infty] ; \frac{1}{q}+\frac{1}{q^{\prime}}=1$. In that case, one obtains an estimate with respect to these norms. For details, see [3, Lemma A.9].

Before proving the Lemma 3.2, we note that the corollary below follows directly from Kolmogorov's continuity criterion (see e.g., [21, Theorem I.2.1]).

COROLLARY 3.4. Let the setting be as in Lemma 3.2 and assume in addition that there exists a $\beta>0$ such that $\beta<\eta-\theta-\frac{1}{p}$. Then there exists a modification of the stochastic convolution process $t \mapsto \int_{0}^{t} \Psi(t-s) \Phi(s) \mathrm{d} W_{H}(s)$, which we denote by $\Psi \diamond \Phi$, and a constant $\tilde{C}$ depending only on $\eta, \beta$ and $p$ and $\bar{C}_{p}$, such that

$$
\begin{aligned}
& \|\Psi \diamond \Phi\|_{L^{p}\left(\Omega ; C^{\beta}\left([0, T] ; Y_{2}\right)\right)} \\
& \quad \leq \tilde{C}\|g\|_{L^{1}(0, T)} \sup _{0 \leq t \leq T}\left\|s \mapsto(t-s)^{-\eta} \Phi(s)\right\|_{L^{p}\left(\Omega ; \gamma\left(0, t ; H, Y_{1}\right)\right)} .
\end{aligned}
$$

Proof of Lemma 3.2. By Proposition 2.9 and assumption (10), it follows that

$$
\left\{s^{\theta} \Psi(s): s \in[0, T]\right\}
$$

is $\gamma$-bounded. Thus, by the Kalton-Weis multiplier Theorem (see Proposition 2.10) and the fact that

$$
\begin{aligned}
\sup _{0 \leq t \leq T} \| s & \mapsto(t-s)^{-\theta} \Phi(s) \|_{L^{p}\left(\Omega ; \gamma\left(0, t ; H, Y_{1}\right)\right)} \\
& \leq T^{\eta-\theta} \sup _{0 \leq t \leq T}\left\|s \mapsto(t-s)^{-\eta} \Phi(s)\right\|_{L^{p}\left(\Omega ; \gamma\left(0, t ; H, Y_{1}\right)\right)}<\infty,
\end{aligned}
$$

it follows that $s \mapsto \Psi(t-s) \Phi(s) 1_{[0, t]}(s) \in L^{p}\left(\Omega ; \gamma\left(0, t ; H, Y_{2}\right)\right)$ for all $t \in[0, T]$. By Theorem 2.5 , this process is $L^{p}$-stochastically integrable on $(0, t)$.

Fix $s, t \in[0, T]$. By the triangle inequality, we have

$$
\begin{aligned}
& \left\|\int_{0}^{t} \Psi(t-u) \Phi(u) \mathrm{d} W_{H}(u)-\int_{0}^{s} \Psi(s-u) \Phi(u) \mathrm{d} W_{H}(u)\right\|_{L^{p}\left(\Omega ; Y_{2}\right)} \\
& \leq\left\|\int_{0}^{s}[\Psi(t-u)-\Psi(s-u)] \Phi(u) \mathrm{d} W_{H}(u)\right\|_{L^{p}\left(\Omega ; Y_{2}\right)} \\
& \quad+\left\|\int_{s}^{t} \Psi(t-u) \Phi(u) \mathrm{d} W_{H}(u)\right\|_{L^{p}\left(\Omega ; Y_{2}\right)} \\
& =\left\|\int_{0}^{s} \int_{s-u}^{t-u} \Psi^{\prime}(v) \Phi(u) \mathrm{d} v \mathrm{~d} W_{H}(u)\right\|_{L^{p}\left(\Omega ; Y_{2}\right)} \\
& \quad+\left\|\int_{s}^{t}(t-u)^{-\theta} \int_{0}^{t-u}\left[v^{\theta} \Psi(v)\right]^{\prime} \Phi(u) \mathrm{d} v \mathrm{~d} W_{H}(u)\right\|_{L^{p}\left(\Omega ; Y_{2}\right)} .
\end{aligned}
$$

We now wish to apply the stochastic Fubini theorem (see [3, Lemma 2.9], [17]). Define $\Upsilon:[0, s] \times[0, t] \times \Omega \rightarrow \mathcal{L}(H, Y)$ by $\Upsilon(u, v, \omega)=1_{[s-u, t-u]}(v) \Psi^{\prime}(v) \Phi(u, \omega)$. 
As $\Psi^{\prime}$ is strongly continuous and $\Phi$ is $H$-strongly measurable, we have that $\Upsilon$ is $H$-strongly measurable. Moreover, as $\Phi$ is adapted, it follows that $\Upsilon_{v}:=\Upsilon(\cdot, v)$ is adapted for almost all $v \in[0, t]$. Finally, by assumptions (9) and (10) we have, for all $v \in[0, t]$ almost surely:

$$
\|\Upsilon(\cdot, v)\|_{\gamma\left(0, s ; H, Y_{2}\right)} \leq v^{\eta-\theta} g(v)\left\|u \mapsto(s-u)^{-\eta} \Phi(u)\right\|_{\gamma\left(0, s ; H, Y_{1}\right)},
$$

where we use that $v \geq s-u$ on $\operatorname{supp}(\Upsilon)$. It follows that $\Upsilon \in L^{1}\left(0, t ; \gamma\left(0, s ; H, Y_{2}\right)\right)$.

The stochastic Fubini theorem in combination with estimate (10) now gives the following:

$$
\begin{aligned}
\| & \int_{0}^{s} \int_{s-u}^{t-u} \Psi^{\prime}(v) \Phi(u) \mathrm{d} v \mathrm{~d} W_{H}(u) \|_{L^{p}\left(\Omega ; Y_{2}\right)} \\
= & \left\|\int_{0}^{t} \int_{(s-v)^{+}}^{(t-v) \wedge s} \Psi^{\prime}(v) \Phi(u) \mathrm{d} W_{H}(u) \mathrm{d} v\right\|_{L^{p}\left(\Omega ; Y_{2}\right)} \\
\leq & \int_{0}^{t} v^{-\theta} g(v)\left\|\int_{(s-v)^{+}}^{(t-v) \wedge s} \Phi(u) \mathrm{d} W_{H}(u)\right\|_{L^{p}\left(\Omega ; Y_{1}\right)} \mathrm{d} v \\
\leq & \bar{C}_{p} \int_{0}^{t} v^{-\theta} g(v)\left\|u \mapsto 1_{\left[(s-v)^{+},(t-v) \wedge s\right]}(u) \Phi(u)\right\|_{L^{p}\left(\Omega ; \gamma\left(0, t ; H, Y_{1}\right)\right)} \mathrm{d} v \\
\leq & \bar{C}_{p} \int_{0}^{t} v^{-\theta} g(v)[(t-s) \wedge v]^{\eta} \\
& \times\left\|u \mapsto[((t-v) \wedge s)-u]^{-\eta} \Phi(u)\right\|_{L^{p}\left(\Omega ; \gamma\left(0,((t-v) \wedge s) ; H, Y_{1}\right)\right)} \mathrm{d} v \\
\leq & \bar{C}_{p}(t-s)^{\eta-\theta} \int_{0}^{t} g(v) \mathrm{d} v \sup _{t \in[0, T]}\left\|u \mapsto(t-u)^{-\eta} \Phi(u)\right\|_{L^{p}\left(\Omega ; \gamma\left(0, t, H ; Y_{1}\right)\right)}
\end{aligned}
$$

In the penultimate estimate above, we use that for all $u \in\left[(s-v)^{+},((t-v) \wedge s)\right.$ one has $((t-v) \wedge s)-u \leq((t-s) \wedge v)$. In the final estimate, we use that

$$
\begin{gathered}
\sup _{s \in[0, T]} \sup _{v \in[0, t]}\left\|u \rightarrow[((t-v) \wedge s)-u]^{-\eta} \Phi(u)\right\|_{L^{p}\left(\Omega ; \gamma\left(0,((t-v) \wedge s) ; H, Y_{1}\right)\right)} \\
\leq \sup _{t \in[0, T]}\left\|u \rightarrow(t-u)^{-\eta} \Phi(u)\right\|_{\left.L^{p}\left(\Omega ; \gamma(0, t) ; H, Y_{1}\right)\right)} .
\end{gathered}
$$

For the final term in (11), one may also check that the conditions of the stochastic Fubini hold and thus

$$
\begin{aligned}
& \left\|\int_{s}^{t}(t-u)^{-\theta} \int_{0}^{t-u}\left[v^{\theta} \Psi(v)\right]^{\prime} \Phi(u) \mathrm{d} v \mathrm{~d} W_{H}(u)\right\|_{L^{p}\left(\Omega ; Y_{2}\right)} \\
& \leq \int_{0}^{t-s} g(v)\left\|\int_{s}^{t-v}(t-u)^{-\theta} \Phi(u) \mathrm{d} W_{H}(u)\right\|_{L^{p}\left(\Omega ; Y_{1}\right)} \mathrm{d} v \\
& \leq \bar{C}_{p} \int_{0}^{t-s} g(v)\left\|u \mapsto 1_{[s, t-v]}(u)(t-u)^{-\theta} \Phi(u)\right\|_{L^{p}\left(\Omega ; \gamma\left(0, t ; H, Y_{1}\right)\right)} \mathrm{d} v \\
& \leq \bar{C}_{p}(t-s)^{\eta-\theta}\|g\|_{L^{1}(0, T)} \sup _{t \in[0, T]}\left\|u \mapsto(t-u)^{-\eta} \Phi(u)\right\|_{L^{p}\left(\Omega ; \gamma\left(0, t ; H, Y_{1}\right)\right)} .
\end{aligned}
$$


By inserting the two estimates above in equation (11), we obtain that

$$
\begin{aligned}
& \left\|\int_{0}^{t} \Psi(t-u) \Phi(u) \mathrm{d} W_{H}(u)-\int_{0}^{s} \Psi(s-u) \Phi(u) \mathrm{d} W_{H}(u)\right\|_{L^{p}\left(\Omega ; Y_{2}\right)} \\
& \quad \leq 2 \bar{C}_{p}(t-s)^{\eta-\theta}\|g\|_{L^{1}(0, T)} \sup _{t \in[0, T]}\left\|u \mapsto(t-u)^{-\eta} \Phi(u)\right\|_{L^{p}\left(\Omega ; \gamma\left(0, t ; H, Y_{1}\right)\right)},
\end{aligned}
$$

which completes the proof as $0 \leq s<t \leq T$ where chosen arbitrarily.

Based on the lemma and the corollary above, we obtain the following result for stochastic convolutions in the $V_{\mathrm{c}}^{\alpha, p}$-norm:

PROPOSITION 3.5. Let the setting be as in Lemma 3.2 and assume in addition that $\frac{1}{p}<\eta-\theta$. Let $\alpha \in\left[0, \frac{1}{2}\right)$. Then $\Psi \diamond \Phi \in V_{\mathrm{c}}^{\alpha, p}\left([0, T] \times \Omega ; Y_{2}\right)$. Moreover, there exists a constant $C$ such that for all $T_{0} \in[0, T]$ we have

$$
\begin{aligned}
& \|\Psi \diamond \Phi\|_{V_{\mathrm{c}}^{\alpha, p}\left(\left[0, T_{0}\right] \times \Omega ; Y_{2}\right)} \\
& \quad \leq C\|g\|_{L^{1}(0, T)} \sup _{0 \leq t \leq T_{0}}\left\|s \mapsto(t-s)^{-\eta} \Phi(s)\right\|_{L^{p}\left(\Omega ; \gamma\left(0, t ; H, Y_{1}\right)\right)} .
\end{aligned}
$$

Proof. For the norm estimate in $L^{p}\left(\Omega ; C\left(\left[0, T_{0}\right] ; Y_{2}\right)\right)$, we apply Corollary 3.4 with $\beta>0$ such that $\beta+\theta<\eta-\frac{1}{p}$. For the estimate in the weighted $\gamma$-norm fix $t \in\left[0, T_{0}\right]$. We apply Lemma 3.1 with $\Phi_{1}(u)=(t-u)^{-\eta} \Phi(u) 1_{[0, t)}(u), \Phi_{2}(r)=\left[r^{\theta} \Psi(r)\right]^{\prime}, R=$ $[0, t]$ and

$$
f(r, u)(s)=(t-s)^{-\alpha}(s-u)^{-\theta}(t-u)^{\eta} 1_{[0, s-u)}(r) 1_{[0, s)}(u) 1_{[0, t)}(s) .
$$

From Lemma 3.1, it follows that

$$
\begin{aligned}
\| s & \mapsto(t-s)^{-\alpha} \int_{0}^{s} \Psi(s-u) \Phi(u) \mathrm{d} W_{H}(u) \|_{L^{p}\left(\Omega ; \gamma\left(0, t ; Y_{2}\right)\right)} \\
& \lesssim t^{\frac{1}{2}+\eta-\alpha-\theta}\|g\|_{L^{1}(0, T)}\left\|s \mapsto(t-s)^{-\eta} \Phi(s)\right\|_{L^{p}\left(\Omega ; \gamma\left(0, t ; H, Y_{1}\right)\right)}
\end{aligned}
$$

Taking the supremum over $t \in\left[0, T_{0}\right]$ and using that $\eta-\theta>0$ (whence $T_{0}^{\frac{1}{2}+\eta-\alpha-\theta} \leq$ $T^{\frac{1}{2}+\eta-\alpha-\theta}$ ), we arrive at the desired result.

Let us denote the deterministic convolution of two mappings $\Psi$ and $\Phi$ by $\Psi * \Phi$. Then, we have the following:

PROPOSITION 3.6. Suppose $\Phi \in L^{p}\left(\Omega ; L^{\infty}\left(0, T ; Y_{1}\right)\right)$ for some $p \in[1, \infty)$. Let $\Psi:[0, T] \rightarrow \mathcal{L}\left(Y_{1}, Y_{2}\right)$ be such that $\Psi x$ is continuously differentiable on $(0, T)$ for all $x \in Y_{1}$. Suppose moreover there exists a function $g \in L^{1}(0, T)$ and a constant $\theta \in(0,1)$ such that for all $v \in(0, T)$ we have

$$
v^{\theta}\left\|\Psi^{\prime}(v) x\right\|_{Y_{2}}+\theta v^{\theta-1}\|\Psi(v) x\|_{Y_{2}} \leq g(v)\|x\|_{Y_{1}}, \text { for all } x \in Y_{1} .
$$

Then there exists a constant $C$ such that for all $T_{0} \in[0, T]$ we have, almost surely

$$
\|\Psi * \Phi\|_{C^{1-\theta}\left(\left[0, T_{0}\right] ; Y_{2}\right)} \leq C\|g\|_{L^{1}\left(0, T_{0}\right)}\|\Phi\|_{L^{\infty}\left(0, T_{0} ; Y_{1}\right)} .
$$


The following corollary is obtained from Lemma 2.7 and the Proposition above by taking $\varepsilon=\frac{3}{2}-\frac{1}{\tau}-\theta$ in Lemma 2.7.

COROLLARY 3.7. Let the setting be as in Proposition 3.6. Assume in addition that $Y_{2}$ has type $\tau$, and let $0 \leq \theta<\frac{3}{2}-\frac{1}{\tau}$. Then for $\alpha \in\left[0, \frac{1}{2}\right)$ and $p \in[1, \infty)$ there exists a constant $C$ such that for $T_{0} \in[0, T]$ one has

$$
\|\Psi * \Phi\|_{V_{\mathrm{c}}^{\alpha, p}\left(\left[0, T_{0}\right] \times \Omega ; Y_{2}\right)} \leq C\|g\|_{L^{1}\left(0, T_{0}\right)}\|\Phi\|_{L^{p}\left(\Omega ; L^{\infty}\left(0, T_{0} ; Y_{1}\right)\right)} .
$$

Proof of Proposition 3.6. Observe that we have, for $0 \leq s<t \leq T_{0}$, almost surely,

$$
\begin{aligned}
& \left\|\int_{0}^{t} \Psi(t-u) \Phi(u, \omega) \mathrm{d} u-\int_{0}^{s} \Psi(s-u) \Phi(u, \omega) \mathrm{d} u\right\|_{Y_{2}} \\
& \leq\left\|\int_{0}^{s} \int_{s-u}^{t-u} \Psi^{\prime}(v) \Phi(u, \omega) \mathrm{d} v \mathrm{~d} u\right\|_{Y_{2}} \\
& \quad+\left\|\int_{s}^{t}(t-u)^{-\theta} \int_{0}^{t-u}\left[v^{\theta} \Psi(v)\right]^{\prime} \Phi(u, \omega) \mathrm{d} v \mathrm{~d} u\right\|_{Y_{2}} .
\end{aligned}
$$

Now

$$
\begin{aligned}
\left\|\int_{0}^{s} \int_{s-u}^{t-u} \Psi^{\prime}(v) \Phi(u, \omega) \mathrm{d} v \mathrm{~d} u\right\|_{Y_{2}} \\
\quad \leq \int_{0}^{s} \int_{s-u}^{t-u} v^{-\theta} g(v)\|\Phi(\omega)\|_{Y_{1}} \mathrm{~d} v \mathrm{~d} u \\
\leq\|\Phi(\omega)\|_{L^{\infty}\left(0, t ; Y_{1}\right)} \int_{0}^{s} \int_{s-u}^{t-u}(s-u)^{-\theta} g(v) \mathrm{d} v \mathrm{~d} u \\
\quad=\|\Phi(\omega)\|_{L^{\infty}\left(0, t ; Y_{1}\right)} \int_{0}^{t} \int_{(s-v)^{+}}^{(t-v) \wedge s}(s-u)^{-\theta} \mathrm{d} u g(v) \mathrm{d} v \quad \text { a.s. }
\end{aligned}
$$

As $\theta \in(0,1)$ we have $a^{1-\theta}-b^{1-\theta} \leq(a-b)^{1-\theta}$ for $a \geq b \geq 0$ and thus also

$$
\begin{aligned}
\int_{(s-v)^{+}}^{(t-v) \wedge s}(s-u)^{-\theta} \mathrm{d} u & =(1-\theta)^{-1}\left[(v \wedge s)^{1-\theta}-\left((s+v-t)^{+}\right)^{1-\theta}\right] \\
& \leq(1-\theta)^{-1}\left[(v \wedge s)-(s+v-t)^{+}\right]^{1-\theta} \leq[(t-s) \wedge v]^{1-\theta} .
\end{aligned}
$$

Combining the two equations above we obtain, almost surely:

$$
\left\|\int_{0}^{s} \int_{s-u}^{t-u} \Psi^{\prime}(v) \Phi(u, \omega) \mathrm{d} v \mathrm{~d} u\right\|_{Y_{2}} \leq(t-s)^{1-\theta}\|\Phi(\omega)\|_{L^{\infty}\left(0, t ; Y_{1}\right)} \int_{0}^{t} g(v) \mathrm{d} v,
$$

Furthermore, almost surely we have

$$
\begin{aligned}
& \left\|\int_{s}^{t}(t-u)^{-\theta} \int_{0}^{t-u}\left[v^{\theta} \Psi(v)\right]^{\prime} \Phi(u, \omega) \mathrm{d} v \mathrm{~d} u\right\|_{Y_{2}} \\
& \quad \leq(1-\theta)^{-1}(t-s)^{1-\theta} \int_{0}^{t} g(v) \mathrm{d} v\|\Phi(\omega)\|_{L^{\infty}\left(0, T ; Y_{1}\right)} .
\end{aligned}
$$

Inserting these two estimates in (13) completes the proof. 


\section{A perturbation result}

In this section, we state and prove the perturbation theorem announced in the introduction, Theorem 4.3 below. In the next subsection, we state the setting and the main result.

\subsection{Setting and main result}

Let $X$ be a Banach space. Let $H$ be a Hilbert space and let $W_{H}$ be an $H$-cylindrical Brownian motion on a probability space $\left(\Omega,\left(\mathcal{F}_{t}\right)_{t \geq 0}, \mathbb{P}\right)$. Consider the following stochastic differential equation set in $X$ :

$$
\left\{\begin{aligned}
\mathrm{d} U(t) & =A U(t) \mathrm{d} t+F(t, U(t)) \mathrm{d} t+G(t, U(t)) \mathrm{d} W_{H}(t) ; \quad t \in[0, T], \\
U(0) & =x_{0},
\end{aligned}\right.
$$

where $T>0, x_{0} \in L^{0}\left(\Omega, \mathcal{F}_{0} ; X\right)$, and $X, A, F$ and $G$ are assumed to satisfy conditions $(\mathbf{X}),(\mathbf{A}),(\mathbf{F})$, and $(\mathbf{G})$ below.

(X) $X$ is a UMD Banach space.

(A) $A$ generates an analytic $C_{0}$-semigroup $S$ on $X$.

(F) For some $\theta_{F}>-1+\left(\frac{1}{\tau}-\frac{1}{2}\right)$, where $\tau \in[1,2]$ is the type of $X$, the function $F:[0, T] \times X \rightarrow X_{\theta_{F}}^{A}$ is measurable in the sense that for all $x \in X$ the mapping $F(\cdot, x):[0, T] \rightarrow X_{\theta_{F}}^{A}$ is strongly measurable.

Moreover, $F$ is uniformly Lipschitz continuous and uniformly of linear growth on $X$. That is to say, there exist constants $C_{0}$ and $C_{1}$ such that for all $t \in[0, T]$ and all $x, y \in X$ we have

$$
\begin{aligned}
\|F(t, x)-F(t, y)\|_{X_{\theta_{F}}^{A}} & \leq C_{0}\|x-y\|_{X}, \\
\|F(t, x)\|_{X_{\theta_{F}}^{A}} & \leq C_{1}\left(1+\|x\|_{X}\right) .
\end{aligned}
$$

(G) For some $\theta_{G}>-\frac{1}{2}$, the function $G:[0, T] \times X \rightarrow \mathcal{L}\left(H, X_{\theta_{G}}^{A}\right)$ is measurable in the sense that for all $h \in H$ and all $x \in X$ the mapping $G(\cdot, x) h:[0, T] \rightarrow X_{\theta_{G}}^{A}$ is strongly measurable.

Moreover, $G$ is uniformly $L_{\gamma}^{2}$-Lipschitz continuous and uniformly of linear growth on $X$. That is to say, there exist constants $C_{0}$ and $C_{1}$ such that for all $\alpha \in\left[0, \frac{1}{2}\right)$, all $t \in[0, T]$, and all simple functions $\phi_{1}, \phi_{2}, \phi:[0, T] \rightarrow X$ one has

$$
\begin{aligned}
& \| s \mapsto(t-s)^{-\alpha}\left[G\left(s, \phi_{1}(s)\right)-G\left(s, \phi_{2}(s)\right)\right] \|_{\gamma\left(0, t ; H, X_{\theta_{G}}^{A}\right)} \\
& \leq C_{0}\left\|s \mapsto(t-s)^{-\alpha}\left[\phi_{1}-\phi_{2}\right]\right\|_{L^{2}(0, t ; X) \cap \gamma(0, t ; X)} ; \\
& \| s \mapsto(t-s)^{-\alpha} G(s, \phi(s)) \|_{\gamma\left(0, t ; H, X_{\theta_{G}}^{A}\right)} \\
& \leq C_{1}\left(1+\left\|s \mapsto(t-s)^{-\alpha} \phi(s)\right\|_{L^{2}(0, t ; X) \cap \gamma(0, t ; X)}\right) .
\end{aligned}
$$

If $Y_{2}$ is a type 2 space and $G:[0, T] \times Y_{1} \rightarrow \gamma\left(H, Y_{2}\right)$ is Lipschitz continuous, uniformly in [0,T], then $G$ is $L_{\gamma}^{2}$-Lipschitz continuous (see [19, Lemma 5.2]). More examples of $L_{\gamma}^{2}$-Lipschitz continuous operators can be found in [19]. 
The following existence and uniqueness result for solutions to equation (SDE) are presented in [19, Theorem 6.2].

THEOREM 4.1. (Van Neerven, Veraar and Weis, 2008) Consider the stochastic differential equation (SDE) in a Banach space $X$, under the assumptions (X), (A), $(\mathbf{F})$, and $(\mathbf{G})$. Let $x_{0} \in L^{p}\left(\Omega, \mathcal{F}_{0} ; X_{\eta}^{A}\right)$ for $p \in(2, \infty)$ and $\eta \geq 0$ satisfying

$$
\eta<\min \left\{\frac{3}{2}-\frac{1}{\tau}+\theta_{F}, \frac{1}{2}-\frac{1}{p}+\theta_{G}\right\} .
$$

Then for any $T>0$ and any $\alpha \in\left[0, \frac{1}{2}\right)$ there exists a unique $U \in V_{\mathrm{c}}^{\alpha, p}([0, T] \times$ $\left.\Omega ; X_{\eta}^{A}\right)$ such that $s \mapsto S(t-s) G(s, U(s)) 1_{[0, t)}(s)$ is $L^{p}$-stochastically integrable for all $t \in[0, T]$, and $U$ satisfies

$$
U(t)=S(t) x_{0}+\int_{0}^{t} S(t-s) F(s, U(s)) d s+\int_{0}^{t} S(t-s) G(s, U(s)) \mathrm{d} W_{H}(s)
$$

almost surely for all $t \in[0, T]$. Moreover

$$
\|U\|_{V_{\mathrm{c}}^{\alpha, p}\left([0, T] \times \Omega ; X_{\eta}^{A}\right)} \lesssim 1+\left\|x_{0}\right\|_{L^{p}\left(\Omega ; X_{\eta}^{A}\right)} .
$$

REMARK 4.2. In [19], the authors assume $\theta_{F} \leq 0$ and $\theta_{G} \leq 0$ (and refer to these constants as $-\theta_{F}$ and $-\theta_{B}$ ). However, one may check that the theorem remains valid for $\theta_{F}, \theta_{G} \geq 0$, which leads to extra space regularity of the solution (i.e., greater values for $\eta$ in (15)). Moreover, in [19], the authors assume $\alpha>\frac{1}{p}-\theta_{G}$. This assumption can be omitted (see [3, Appendix A.3]).

Keeping in mind possible applications in approximations of solutions to stochastic partial differential equations, we now introduce a space $X_{0}$, and an operator $A_{0}$ satisfying the following conditions:

$\left(\mathbf{X}_{0}\right) X_{0}$ is a (possibly finite dimensional) closed subspace of $X$, and there exists a bounded projection $P_{0}: X \rightarrow X_{0}$ such that $P_{0}(X)=X_{0}$.

$\left(\mathbf{A}_{0}\right) A_{0}$ is the generator of an analytic $C_{0}$-semigroup $S_{0}$ on $X_{0}$, and $\omega \geq 0, \theta \in\left(0, \frac{\pi}{2}\right]$ and $K>0$ are such that $A$ and $A_{0}$ are both of type $(\omega, \theta, K)$.

Let $i_{X_{0}}$ represent the canonical embedding of $X_{0}$ into $X$ (we omit $i_{X_{0}}$ when its use is clear from the context). For $t \geq 0$, define $\tilde{S}_{0}(t) \in \mathcal{L}(X)$ by $\tilde{S}_{0}(t):=i_{X_{0}} S_{0}(t) P_{0}$, this defines a degenerate $C_{0}$-semigroup, i.e., $\tilde{S}_{0}$ satisfies the semigroup property but $\tilde{S}_{0}(0)=i_{X_{0}} P_{0}$ (which is clearly not the identity unless $X_{0}=X$ ).

In order to avoid technical difficulties later on, we from now on define the spaces $X_{\beta}^{A}, \beta \in \mathbb{R}$, in terms of $((\omega+1) I-A)^{\beta}$. Once this is fixed, it makes sense to define $\operatorname{Lip}(F)$ and $M(F)$ to be the least constants $C_{0}$ and $C_{1}$ for which the Lipschitz and linear growth conditions of (F) hold. Similarly, we define $\operatorname{Lip}_{\gamma}(G)$ and $M(G)$ to be the least constants $C_{0}$ and $C_{1}$ for which the Lipschitz and linear growth conditions of (G) hold.

THEOREM 4.3. Consider the stochastic differential equation (SDE) in a Banach space $X$, under the assumptions $(\mathbf{X}),(\mathbf{A}),(\mathbf{F})$, and $(\mathbf{G})$. Let $X_{0}$ and $A_{0}$ satisfy $\left(\mathbf{X}_{0}\right)$ and $\left(\mathbf{A}_{0}\right)$. Suppose there exist $\delta \in[0,1]$ and $p \in(2, \infty)$ satisfying 


$$
0 \leq \delta<\min \left\{\frac{3}{2}-\frac{1}{\tau}+\theta_{F}, \frac{1}{2}-\frac{1}{p}+\theta_{G}\right\}
$$

such that for some $\lambda_{0} \in \mathbb{C}$ such that $\Re e\left(\lambda_{0}\right) \geq \omega$ we have

$$
D_{\delta}\left(A, A_{0}\right):=\left\|R\left(\lambda_{0}: A\right)-i_{X_{0}} R\left(\lambda_{0}: A_{0}\right) P_{0}\right\|_{\mathcal{L}\left(X_{\delta-1}^{A}, X\right)}<\infty .
$$

Suppose $x_{0} \in L^{p}\left(\Omega, \mathcal{F}_{0} ; X_{\delta}^{A}\right)$ and $y_{0} \in L^{p}\left(\Omega, \mathcal{F}_{0} ; X\right)$.

Then for any $\alpha \in\left[0, \frac{1}{2}\right)$ there exists a unique process $U^{(0)} \in V_{\mathrm{c}}^{\alpha, p}\left([0, T] \times \Omega ; X_{0}\right)$ such that $s \mapsto 1_{[0, t]}(s) S_{0}(t-s) P_{0} G\left(s, U^{(0)}(s)\right)$ is $L^{p}$-stochastically integrable for all $t \in[0, T]$, and for all $t \in[0, T]$ we have

$$
\begin{aligned}
U^{(0)}(t)= & S_{0}(t-s) P_{0} y_{0}+\int_{0}^{T} S_{0}(t-s) P_{0} F\left(s, U^{(0)}(s)\right) d s \\
& +\int_{0}^{t} S_{0}(t-s) P_{0} G\left(s, U^{(0)}(s)\right) \mathrm{d} W_{H}(s), \quad \text { a.s. }
\end{aligned}
$$

Moreover,

$$
\begin{aligned}
& \left\|U-i_{X_{0}} U^{(0)}\right\|_{V_{\mathrm{c}}^{\alpha, p}([0, T] \times \Omega ; X)} \\
& \quad \lesssim\left\|x_{0}-y_{0}\right\|_{L^{p}(\Omega ; X)}+D_{\delta}\left(A, A_{0}\right)\left(1+\left\|x_{0}\right\|_{L^{p}\left(\Omega ; X_{\delta}^{A}\right)}\right) .
\end{aligned}
$$

The implied constant depends on $X_{0}$ only in terms of $\left\|P_{0}\right\|_{\mathcal{L}\left(X, X_{0}\right)}$, on $A$ and $A_{0}$ only in terms of $1 \vee D_{\delta}\left(A, A_{0}\right), \omega, \theta$ and $K$, and on $F$ and $G$ only in terms of their Lipschitz. and linear growth constants $\operatorname{Lip}(F), \operatorname{Lip}_{\gamma}(G), M(F)$ and $M_{\gamma}(G)$.

\subsection{Proof of main result}

To prove Theorem 4.3, we need a proposition concerning the $\gamma$-boundedness of $S-\tilde{S}_{0}$. The proof of this proposition is postponed to the end of this Section.

PROPOSITION 4.4. Let $A, A_{0}$ be as introduced above, i.e., A generates an analytic $C_{0}$-semigroup on $X$ and $A_{0}$ generates an analytic $C_{0}$-semigroup on $X_{0}$, and $\omega \geq 0, \theta \in\left(0, \frac{\pi}{2}\right]$ and $K>0$ are such that $A$ and $A_{0}$ are of type $(\omega, \theta, K)$. Suppose there exists a $\lambda_{0} \in \mathbb{C}$, 祉 $\left(\lambda_{0}\right)>\omega$, and $\delta \in \mathbb{R}$ such that $D_{\delta}\left(A, A_{0}\right)<\infty$, where $D_{\delta}\left(A, A_{0}\right)$ is as defined in (17). Set

$$
\omega^{\prime}=(\omega+1)(\cos \theta)^{-1} .
$$

Then for all $\beta \in \mathbb{R}$ such that $\beta \in[\delta-1, \delta]$ one has

$$
\sup _{t \in[0, \infty)} t^{\delta-\beta} e^{-\omega^{\prime} t}\left\|S(t)-\tilde{S}_{0}(t)\right\|_{\mathcal{L}\left(X_{\beta}^{A}, X\right)} \lesssim D_{\delta}\left(A, A_{0}\right),
$$

and

$$
\sup _{t \in(0, \infty)} t^{\delta-\beta+1} e^{-\omega^{\prime} t}\left\|S^{\prime}(t)-\tilde{S}_{0}^{\prime}(t)\right\|_{\mathcal{L}\left(X_{\beta}^{A}, X\right)} \lesssim D_{\delta}\left(A, A_{0}\right)
$$

with implied constants depending only on $\left\|P_{0}\right\|_{\mathcal{L}\left(X, X_{0}\right)}, \omega, \theta, K$, and $\delta-\beta$. 
Moreover, for all $\alpha>\delta-\beta$ we have, for $t \in[0, T]$,

$$
\gamma_{\left[X_{\beta}^{A}, X\right]}\left(\left\{s^{\alpha}\left[S(s)-\tilde{S}_{0}(s)\right] ; 0 \leq s \leq t\right\}\right) \lesssim t^{\alpha+\beta-\delta} D_{\delta}\left(A, A_{0}\right),
$$

with implied constant depending only on $\left\|P_{0}\right\|_{\mathcal{L}\left(X, X_{0}\right)}, \omega, \theta, K, \delta-\beta, \alpha$, and $T$.

Proof of Theorem 4.3. We split the proof into several parts.

Part 1.

In order to prove existence and uniqueness of $U^{(0)} \in V_{\mathrm{c}}^{\alpha, p}\left([0, T] \times \Omega ; X_{0}\right)$ satisfying (18) it suffices, by Theorem 4.1, to prove that there exist $\eta_{F}>-\frac{3}{2}+\frac{1}{\tau}$ and $\eta_{G}>-\frac{1}{2}+\frac{1}{p}$ such that $P_{0} F:[0, T] \times X \rightarrow X_{0, \eta_{F}}^{A_{0}}$ is Lipschitz continuous and of linear growth and $P_{0} G:[0, T] \times X \rightarrow \gamma\left(H, X_{0, \eta_{G}}^{A_{0}}\right)$ is $L_{\gamma}^{2}$-Lipschitz continuous and of linear growth. If $\theta_{F} \geq 0$, then clearly we may take $\eta_{F}=0$, and we have $\operatorname{Lip}\left(P_{0} F\right) \leq\left\|P_{0}\right\|_{\mathcal{L}\left(X, X_{0}\right)} \operatorname{Lip}(F), M\left(P_{0} F\right) \leq\left\|P_{0}\right\|_{\mathcal{L}\left(X, X_{0}\right)} M(F)$. The same goes for $\theta_{G} \geq 0$.

Now, suppose $\theta_{F}<0$. Let $\bar{\omega}>\omega^{\prime}$, where $\omega^{\prime}$ is as in Proposition 4.4. From the representation of negative fractional powers of an operator $A$ generating an analytic $C_{0}$-semigroup $S$ of type $(\omega, \theta, K)$ (see [20, Chapter 2.6]) and Proposition 4.4, it follows that for $\beta \in[\delta-1, \delta], \eta<\beta-\delta$, and $x \in X$ we have

$$
\begin{aligned}
& \left\|P_{0} x\right\|_{X_{0, \eta}^{A_{0}}} \approx\left\|\left(\bar{\omega} I-A_{0}\right)^{\eta} P_{0} x\right\|_{X}=\left\|\frac{1}{\Gamma(-\eta)} \int_{0}^{\infty} t^{-\eta-1} e^{-\bar{\omega} t} \tilde{S}_{0}(t) x \mathrm{~d} t\right\|_{X} \\
& \leq \frac{1}{\Gamma(-\eta)} \int_{0}^{\infty} t^{-\eta-1} e^{-\bar{\omega} t}\left\|\left(S(t)-\tilde{S}_{0}(t)\right) x\right\|_{X} \mathrm{~d} t+\frac{1}{\Gamma(-\eta)}\left\|\int_{0}^{\infty} t^{-\eta-1} e^{-\bar{\omega} t} S(t) x \mathrm{~d} t\right\|_{X} \\
& \lesssim D_{\delta}\left(A, A_{0}\right) \int_{0}^{\infty} t^{-\eta-1+\beta-\delta} e^{-\left(\bar{\omega}-\omega^{\prime}\right) t} \mathrm{~d} t\|x\|_{X_{\beta}^{A}}+\left\|(\bar{\omega} I-A)^{\eta} x\right\|_{X},
\end{aligned}
$$

with implied constants depending on $X_{0}$ only in terms of $\left\|P_{0}\right\|_{\mathcal{L}\left(X, X_{0}\right)}$ and on $A$ and $A_{0}$ only in terms of $\omega, \theta$, and $K$. Thus, for $\beta \in[\delta-1, \delta], \eta<\beta-\delta$ we have

$$
\begin{aligned}
& \left\|P_{0} x\right\|_{X_{0, \eta}^{A_{0}}} \bar{\sim}\left\|\left(\bar{\omega} I-A_{0}\right)^{\eta} P_{0} x\right\|_{X} \\
& \quad \lesssim\left(1+D_{\delta}\left(A, A_{0}\right)\right)\left\|(\bar{\omega} I-A)^{\beta} x\right\|_{X} \bar{\sim}\left(1+D_{\delta}\left(A, A_{0}\right)\right)\|x\|_{X_{\beta}^{A}},
\end{aligned}
$$

with implied constants depending on $X_{0}$ only in terms of $\left\|P_{0}\right\|_{\mathcal{L}\left(X, X_{0}\right)}$ and on $A$ and $A_{0}$ only in terms of $\omega, \theta$, and $K$.

By assumption (16), we have $\theta_{F}>-\frac{3}{2}+\frac{1}{\tau}+\delta \geq \delta-1$. Hence, one can pick $\eta_{F}$ such that

$$
-\frac{3}{2}+\frac{1}{\tau}<\eta_{F}<\theta_{F}-\delta .
$$

On the other hand, we have $\theta_{F}<0 \leq \delta$. Thus, by (22) with $\beta=\theta_{F}$ and $\eta=\eta_{F}$ it follows that $P_{0} F:[0, T] \times X \rightarrow X_{0, \eta_{F}}^{A_{0}}$ is Lipschitz continuous and

$$
\operatorname{Lip}\left(P_{0} F\right) \lesssim\left(1+D_{\delta}\left(A, A_{0}\right)\right) \operatorname{Lip}(F) ; M\left(P_{0} F\right) \lesssim\left(1+D_{\delta}\left(A, A_{0}\right)\right) M(F),
$$


with implied constant depending on $X_{0}$ only in terms of $\left\|P_{0}\right\|_{\mathcal{L}\left(X, X_{0}\right)}$ and on $A$ and $A_{0}$ only in terms of $\omega, \theta$, and $K$.

Similarly, if $\theta_{G}<0$ there exists a $\eta_{G}$ such that

$$
-\frac{1}{2}+\frac{1}{p}<\eta_{G}<\theta_{G}-\delta
$$

such that $P_{0} G:[0, T] \times X \rightarrow \gamma\left(H, X_{0, \eta_{G}}^{A_{0}}\right)$ is $L_{\gamma}^{2}$-Lipschitz continuous and

$$
\operatorname{Lip}_{\gamma}\left(P_{0} G\right) \lesssim\left(1+D_{\delta}\left(A, A_{0}\right)\right) \operatorname{Lip}_{\gamma}(G) ; M_{\gamma}\left(P_{0} G\right) \lesssim\left(1+D_{\delta}\left(A, A_{0}\right)\right) M_{\gamma}(G),
$$

with implied constant depending on $X_{0}$ only in terms of $\left\|P_{0}\right\|_{\mathcal{L}\left(X, X_{0}\right)}$ and on $A$ and $A_{0}$ only in terms of $\omega, \theta$, and $K$.

In conclusion, we are now in the position to apply Theorem 4.1 with $\theta_{F}$ and $\theta_{G}$ in that theorem chosen to be $\eta_{F}$ and $\eta_{G}$, and thus obtain existence of a unique process $U^{(0)} \in V_{\mathrm{c}}^{\alpha, p}\left(\left[0, T_{0}\right] \times \Omega ; X_{0}\right), \alpha \in\left[0, \frac{1}{2}\right)$, satisfying (18). Note that we have chosen $\eta_{F}$ and $\eta_{G}$ such that they are non-positive.

\section{Part 2.}

Define $\tilde{U}^{(0)}=i_{X_{0}} U^{(0)}$ and observe that if $U^{(0)}$ satisfies (18), then $\tilde{U}^{(0)}$ satisfies

$$
\begin{aligned}
\tilde{U}^{(0)}(t)= & \tilde{S}_{0}(t-s) y_{0}+\int_{0}^{T} \tilde{S}_{0}(t-s) F\left(s, \tilde{U}^{(0)}(s)\right) d s \\
& +\int_{0}^{t} \tilde{S}_{0}(t-s) G\left(s, \tilde{U}^{(0)}(s)\right) \mathrm{d} W_{H}(s), \quad \text { a.s. }
\end{aligned}
$$

Let $T_{0} \in[0, T]$ be fixed. By the above we have

$$
\begin{aligned}
\| U & -\tilde{U}^{(0)} \|_{V_{\mathrm{c}}^{\alpha, p}\left(\left[0, T_{0}\right] \times \Omega ; X\right)} \\
\leq & \left\|\left(S-\tilde{S}_{0}\right) x_{0}\right\|_{V_{\mathrm{c}}^{\alpha, p}\left(\left[0, T_{0}\right] \times \Omega ; X\right)}+\left\|\tilde{S}_{0}\left(x_{0}-y_{0}\right)\right\|_{V_{\mathrm{c}}^{\alpha, p}\left(\left[0, T_{0}\right] \times \Omega ; X\right)} \\
& +\left\|t \mapsto \int_{0}^{t} \tilde{S}_{0}(t-s)\left[F(s, U(s))-F\left(s, \tilde{U}^{(0)}(s)\right)\right] d s\right\|_{V_{\mathrm{c}}^{\alpha, p}\left(\left[0, T_{0}\right] \times \Omega ; X\right)} \\
& +\left\|t \mapsto \int_{0}^{t}\left[S(t-s)-\tilde{S}_{0}(t-s)\right] F(s, U(s)) d s\right\|_{V_{\mathrm{c}}^{\alpha, p}\left(\left[0, T_{0}\right] \times \Omega ; X\right)} \\
& +\left\|t \mapsto \int_{0}^{t} \tilde{S}_{0}(t-s)\left[G(s, U(s))-G\left(s, \tilde{U}^{(0)}(s)\right)\right] \mathrm{d} W_{H}(s)\right\|_{V_{\mathrm{c}}^{\alpha, p}\left(\left[0, T_{0}\right] \times \Omega ; X\right)} \\
& +\left\|t \mapsto \int_{0}^{t}\left[S(t-s)-\tilde{S}_{0}(t-s)\right] G(s, U(s)) \mathrm{d} W_{H}(s)\right\|_{V_{\mathrm{c}}^{\alpha, p}\left(\left[0, T_{0}\right] \times \Omega ; X\right)} .
\end{aligned}
$$

Let $\eta_{F}$ and $\eta_{G}$ be as defined in part 1 . Let $\varepsilon>0$ be such that

$$
\begin{aligned}
& \varepsilon \leq 1-2 \alpha \\
& \varepsilon<\min \left\{\frac{3}{2}-\frac{1}{\tau}+\eta_{F}, \frac{1}{2}-\frac{1}{p}+\eta_{G}\right\} .
\end{aligned}
$$


It follows that $\varepsilon+\delta<\min \left\{\frac{3}{2}-\frac{1}{\tau}+\theta_{F}, \frac{1}{2}-\frac{1}{p}+\theta_{G}\right\}$. By inequality (7) we may assume, without loss of generality, that $\alpha=\frac{1}{2}-\varepsilon / 2$.

We estimate each of the six terms on the right-hand side of (25) in parts $2 \mathrm{a}-2 \mathrm{f}$ below. In part $2 \mathrm{c}$ and $2 \mathrm{e}$, we keep track of the dependence on $T_{0}$, for the other parts this is not necessary.

Part $2 a$.

By Proposition 4.4 with $\beta=\delta$ and $\alpha=\varepsilon / 2$ there exists an $\mathcal{M}>0$ depending on $X_{0}$ only in terms of $\left\|P_{0}\right\|_{\mathcal{L}\left(X, X_{0}\right)}$, and on $A$ and $A_{0}$ only in terms om $\omega, \theta$ and $K$, such that

$$
\begin{array}{r}
\sup _{t \in\left[0, T_{0}\right]}\left\|S(t)-\tilde{S}_{0}(t)\right\|_{\mathcal{L}\left(X_{\delta}^{A}, X\right)} \leq \mathcal{M} D_{\delta}\left(A, A_{0}\right) ; \\
\gamma_{\left[X_{\delta}^{A}, X\right]}\left\{t^{\varepsilon / 2}\left(S(t)-\tilde{S}_{0}(t)\right): t \in\left[0, T_{0}\right]\right\} \leq \mathcal{M} D_{\delta}\left(A, A_{0}\right) .
\end{array}
$$

Thus, by Proposition 2.10, we have

$$
\begin{aligned}
& \left\|\left(S-\tilde{S}_{0}\right) x_{0}\right\|_{V_{\mathrm{c}}^{\alpha, p}\left(\left[0, T_{0}\right] \times \Omega ; X\right)} \\
& \quad \leq \mathcal{M} D_{\delta}\left(A, A_{0}\right)\left[\sup _{t \in\left[0, T_{0}\right]}\left\|s \mapsto(t-s)^{-\alpha} s^{-\varepsilon / 2} x_{0}\right\|_{L^{p}\left(\Omega ; \gamma\left(0, t ; X_{\delta}^{A}\right)\right)}+\left\|x_{0}\right\|_{L^{p}\left(\Omega ; X_{\delta}^{A}\right)}\right] .
\end{aligned}
$$

For $f \in L^{2}(0, t)$ and $x \in L^{p}\left(\Omega ; X_{\delta}^{A}\right)$ we have

$$
\|f \otimes x\|_{L^{p}\left(\Omega ; \gamma\left(0, t ; X_{\delta}^{A}\right)\right)}=\|f\|_{L^{2}(0, t)}\|x\|_{L^{p}\left(\Omega ; X_{\delta}^{A}\right)} .
$$

Thus, recalling that $\alpha=\frac{1}{2}-\varepsilon / 2$, we have

$$
\begin{aligned}
\sup _{t \in\left[0, T_{0}\right]} \| & \mapsto \mapsto(t-s)^{-\alpha} s^{-\varepsilon / 2} x_{0} \|_{L^{p}\left(\Omega ; \gamma\left(0, t ; X_{\delta}^{A}\right)\right)} \\
& \leq T^{\frac{1}{2}-\alpha-\varepsilon / 2}\left\|s \mapsto(1-s)^{-\alpha} s^{-\varepsilon / 2}\right\|_{L^{2}(0,1)}\left\|x_{0}\right\|_{L^{p}\left(\Omega ; X_{\delta}^{A}\right)} \\
& \leq C_{\varepsilon} T^{\frac{1}{2}-\alpha-\varepsilon / 2}\left\|x_{0}\right\|_{L^{p}\left(\Omega ; X_{\delta}^{A}\right)},
\end{aligned}
$$

where $C_{\varepsilon}$ is a constant depending only on $\varepsilon$. Hence

$$
\left\|\left(S-\tilde{S}_{0}\right) x_{0}\right\|_{V_{\mathrm{c}}^{\alpha, p}\left(\left[0, T_{0}\right] \times \Omega ; X\right)} \leq \mathcal{M} D_{\delta}\left(A, A_{0}\right)\left(1+C_{\varepsilon} T^{\frac{1}{2}-\alpha-\varepsilon / 2}\right)\left\|x_{0}\right\|_{L^{p}\left(\Omega ; X_{\delta}^{A}\right)} .
$$

\section{Part $2 b$.}

By assumption (see Remark 2.4) there exists an $\mathcal{M}$ depending only on $\left\|P_{0}\right\|_{\mathcal{L}\left(X, X_{0}\right)}, \omega, \theta$, and $K$ and $T$ such that we have that $\sup _{t \in[0, T]}\left\|\tilde{S}_{0}(t)\right\|_{\mathcal{L}\left(X, X_{0}\right)} \leq$ $\mathcal{M}$. Moreover, by Lemma 2.11 we may pick $\mathcal{M}$ such that in addition we have that $\gamma_{[X, X]}\left\{t^{\varepsilon / 2} \tilde{S}_{0}(t): t \in\left[0, T_{0}\right]\right\} \leq \mathcal{M}$. Thus, by the same argument as in part 2 a, we have

$$
\left\|\tilde{S}_{0}\left(x_{0}-y_{0}\right)\right\|_{V_{\mathrm{c}}^{\alpha, p}\left(\left[0, T_{0}\right] \times \Omega ; X\right)} \leq \mathcal{M}\left(1+C_{\varepsilon} T^{\frac{1}{2}-\alpha-\varepsilon / 2}\right)\left\|x_{0}-y_{0}\right\|_{L^{p}(\Omega ; X)} .
$$




\section{Part 2c.}

Recall that in part 1 , we chose $\eta_{F}$ such that $\eta_{F} \leq 0$. By inequality (3), there exists an $\mathcal{M}$ depending only on $\omega, \theta, K$ and $T$ such that for all $t \in[0, T]$ we have

$$
t^{-\eta_{F}+\varepsilon}\left\|S_{0}^{\prime}(t)\right\|_{\mathcal{L}\left(X_{0, \eta_{F}}^{A_{0}}, X_{0}\right)}+\left(\varepsilon-\eta_{F}\right) t^{-\eta_{F}+\varepsilon-1}\left\|S_{0}(t)\right\|_{\mathcal{L}\left(X_{0, \eta_{F}}^{A_{0}}, X_{0}\right)} \leq \mathcal{M} t^{-1+\varepsilon} .
$$

By Corollary 3.7 with $Y_{1}=X_{0, \eta_{F}}^{A_{0}}, Y_{2}=X$,

$$
\begin{gathered}
\Phi(s)=P_{0}\left[F(s, U(s))-F\left(s, \tilde{U}^{(0)}(s)\right)\right], \\
\Psi(s)=S_{0}(s), \theta=-\eta_{F}+\varepsilon \text { and } g(v)=\mathcal{M} v^{-1+\varepsilon}, \text { it follows that } \\
\left\|t \mapsto \int_{0}^{t} \tilde{S}_{0}(t-s)\left[F(s, U(s))-F\left(s, \tilde{U}^{(0)}(s)\right)\right] d s\right\|_{V_{\mathrm{c}}^{\alpha, p}\left(\left[0, T_{0}\right] \times \Omega ; X\right)} \\
\lesssim T_{0}^{\varepsilon}\left\|P_{0}\left[F(\cdot, U)-F\left(\cdot, \tilde{U}^{(0)}\right)\right]\right\|_{L^{p}\left(\Omega ; L^{\infty}\left(0, T_{0} ; X_{0, \eta_{F}}^{A_{0}}\right)\right)} \\
\leq T_{0}^{\varepsilon} \operatorname{Lip}\left(P_{0} F\right)\left\|U-\tilde{U}^{(0)}\right\|_{L^{p}\left(\Omega ; L^{\infty}\left(0, T_{0} ; X\right)\right)} \\
\lesssim T_{0}^{\varepsilon}\left(1+D_{\delta}\left(A, A_{0}\right)\right) \operatorname{Lip}(F)\left\|U-\tilde{U}^{(0)}\right\|_{L^{p}\left(\Omega ; L^{\infty}\left(0, T_{0} ; X\right)\right)},
\end{gathered}
$$

where the second last estimate follows by Lipschitz continuity of $P_{0} F$, and the final estimate follows by (23). Note that the implied constants are independent of $T_{0}$ and depend on $X_{0}$ only in terms of $\left\|P_{0}\right\|_{\mathcal{L}\left(X, X_{0}\right)}$ and on $A_{0}$ only in terms of $\omega, \theta$, and $K$.

Part $2 d$.

For brevity set $\zeta=\left(\delta-\theta_{F}\right)^{+}+\varepsilon$. By assumption (16), we have $\theta_{F} \wedge \delta \in[\delta-1, \delta]$, and thus, we may apply Proposition 4.4 with $\beta=\theta_{F} \wedge \delta$ to obtain that there exists a constant $\mathcal{M}$ depending only on $\left\|P_{0}\right\|_{\mathcal{L}\left(X, X_{0}\right)}, \omega, \theta, K,\left(\delta-\theta_{F}\right)^{+}$and $T$ such that for all $t \in[0, T]$ we have

$$
\begin{aligned}
& t^{\zeta}\left\|S^{\prime}(t)-\tilde{S}_{0}^{\prime}(t)\right\|_{\mathcal{L}\left(X_{\theta_{F} \wedge \delta}^{A}, X\right)}+\zeta t^{\zeta-1}\left\|S(t)-\tilde{S}_{0}(t)\right\|_{\mathcal{L}\left(X_{\theta_{F} \wedge \delta}^{A}, X\right)} \\
& \quad \leq \mathcal{M} D_{\delta}\left(A, A_{0}\right) t^{-1+\varepsilon} .
\end{aligned}
$$

Thus by Corollary 3.7 with $Y_{1}=X_{\theta_{F} \wedge \delta}^{A}, Y_{2}=X, \Phi(s)=F(s, U(s)), \Psi(s)=$ $S(s)-\tilde{S}_{0}(s), \theta=\zeta=\left(\delta-\theta_{F}\right)^{+}+\varepsilon, g(v)=\mathcal{M} D_{\delta}\left(A, A_{0}\right) v^{-1+\varepsilon}$, we obtain

$$
\begin{aligned}
\| t & \mapsto \int_{0}^{t}\left[S(t-s)-\tilde{S}_{0}(t-s)\right] F(s, U(s)) d s \|_{V_{\mathrm{c}}^{\alpha, p}\left(\left[0, T_{0}\right] \times \Omega ; X\right)} \\
& \left.\left.\lesssim D_{\delta}\left(A, A_{0}\right)\|F(\cdot, U)\|_{L^{p}\left(\Omega ; L^{\infty}\left(0, T_{0} ; X_{\theta_{F}}^{A} \delta\right.\right.}\right)\right) \\
& \leq D_{\delta}\left(A, A_{0}\right) M(F)\|U\|_{L^{p}\left(\Omega ; L^{\infty}\left(0, T_{0} ; X\right)\right)} \\
& \lesssim D_{\delta}\left(A, A_{0}\right) M(F)\left(1+\left\|x_{0}\right\|_{L^{p}(\Omega ; X)}\right),
\end{aligned}
$$

where the penultimate estimate follows by the linear growth condition on $F$ and the final estimate by (15). Note that the implied constants are independent of $T_{0}$, and depend on $X_{0}$ only in terms of $\left\|P_{0}\right\|_{\mathcal{L}\left(X, X_{0}\right)}$, and on $A$ and $A_{0}$ only in terms of $\omega, \theta$ and $K$. 


\section{Part 2e.}

Observe that if $G:[0, T] \times X \rightarrow \mathcal{L}\left(H, X_{\theta_{G}}\right)$ satisfies (G) and $\Phi_{1}, \Phi_{2} \in$ $V_{\mathrm{c}}^{\alpha, p}([0, T] \times \Omega ; X)$ for some $p \geq 2$, then

$$
\begin{gathered}
\sup _{0 \leq t \leq T}\left\|s \mapsto(t-s)^{-\alpha}\left[G\left(s, \Phi_{1}(s)\right)-G\left(s, \Phi_{2}(s)\right)\right]\right\|_{L^{p}\left(\Omega ; \gamma\left(0, t ; H, X_{\theta_{G}}^{A}\right)\right)} \\
\leq\left(1+(1-2 \alpha)^{-\frac{1}{2}} T^{\frac{1}{2}-\alpha}\right) \operatorname{Lip}_{\gamma}(G)\left\|\Phi_{1}-\Phi_{2}\right\|_{V_{\mathrm{c}}^{\alpha, p}([0, T] \times \Omega ; X)}
\end{gathered}
$$

Recall that in part 1 , we chose $\eta_{G}$ such that $\eta_{G} \leq 0$. By equation (3), there exists an $\mathcal{M}$ depending only on $\omega, \theta, K$, and $T$ such that for all $t \in[0, T]$ we have

$$
\begin{aligned}
& t^{-\eta_{G}+\varepsilon / 2}\left\|S_{0}^{\prime}(t)\right\|_{\mathcal{L}\left(X_{0,-\eta_{G}}^{A}, X_{0}\right)}+\left(\varepsilon / 2-\eta_{G}\right) t^{-\eta_{G}+\varepsilon / 2-1}\left\|S_{0}(t)\right\|_{\mathcal{L}\left(X_{0,-\eta_{G}}^{A}, X_{0}\right)} \\
& \quad \leq \mathcal{M} t^{-1+\varepsilon / 2} .
\end{aligned}
$$

By applying Proposition 3.5 with $Y_{1}=X_{0, \eta_{G}}^{A_{0}}, Y_{2}=X, \Psi(s)=S_{0}(s), \eta=\alpha$, $\theta=-\eta_{G}+\varepsilon / 2, g(v)=\mathcal{M} v^{-1+\varepsilon / 2}$ and $\Phi(s)=P_{0}\left[G(s, U(s))-G\left(s, \tilde{U}^{(0)}(s)\right)\right]$ we obtain

$$
\begin{aligned}
\| t & \mapsto \int_{0}^{t} \tilde{S}_{0}(t-s)\left[G(s, U(s))-G\left(s, \tilde{U}^{(0)}(s)\right)\right] \mathrm{d} W_{H}(s) \|_{V_{\mathrm{c}}^{\alpha, p}\left(\left[0, T_{0}\right] \times \Omega ; X\right)} \\
& \lesssim T_{0}^{\frac{1}{2} \varepsilon} \sup _{0 \leq t \leq T_{0}}\left\|s \mapsto(t-s)^{-\alpha} P_{0}\left[G(s, U(s))-G\left(s, \tilde{U}^{(0)}(s)\right)\right]\right\|_{L^{p}\left(\Omega ; \gamma\left(0, t, H ; X_{0, \eta_{G}}^{A_{0}}\right)\right)} \\
& \lesssim T_{0}^{\frac{1}{2} \varepsilon}\left(1+D_{\delta}\left(A, A_{0}\right)\right) \operatorname{Lip}_{\gamma}(G)\left\|U-\tilde{U}^{(0)}\right\|_{V_{\mathrm{c}}^{\alpha, p}\left(\left[0, T_{0}\right] \times \Omega ; X\right)},
\end{aligned}
$$

where the final estimate follows from estimates (30) and (24). Note that the implied constants are independent of $T_{0}$ and depend on $X_{0}$ only in terms of $\left\|P_{0}\right\|_{\mathcal{L}\left(X, X_{0}\right)}$, and on $A$ and $A_{0}$ only in terms of $\omega, \theta$, and $K$.

Part $2 f$.

Observe that, for $\Phi \in V_{\mathrm{c}}^{\alpha, p}([0, T] \times \Omega ; X)$, we have

$$
\begin{aligned}
& \sup _{0 \leq t \leq T}\left\|s \mapsto(t-s)^{-\alpha} G(s, \Phi(s))\right\|_{L^{p}\left(\Omega ; \gamma\left(0, t ; H, X_{\theta_{G}}^{A}\right)\right)} \\
& \quad \leq\left(1+(1-2 \alpha)^{-\frac{1}{2}} T^{\frac{1}{2}-\alpha}\right) M_{\gamma}(G)\left(1+\|\Phi\|_{V_{\mathrm{c}}^{\alpha, p}([0, T] \times \Omega ; X)}\right)
\end{aligned}
$$

For brevity set $\zeta=\left(\delta-\theta_{G}\right)^{+}+\varepsilon / 2$. By Proposition 4.4 with $\beta=\theta_{G} \wedge \delta \in[\delta-1, \delta]$, we have that there exists a constant $\mathcal{M}$ depending only on $\left\|P_{0}\right\|_{\mathcal{L}\left(X, X_{0}\right)}, \omega, \theta, K$, $\left(\delta-\theta_{G}\right)^{+}$and $T$ such that for all $t \in[0, T]$ we have

$$
\begin{aligned}
& t^{\zeta}\left\|S^{\prime}(t)-\tilde{S}_{0}^{\prime}(t)\right\|_{\mathcal{L}\left(X_{\theta_{G} \wedge \delta}^{A}, X\right)}+\zeta t^{\zeta-1}\left\|S(t)-\tilde{S}_{0}(t)\right\|_{\mathcal{L}\left(X_{\theta_{G} \wedge \delta}^{A}, X\right)} \\
& \leq \mathcal{M} D_{\delta}\left(A, A_{0}\right) t^{-1+\varepsilon / 2}
\end{aligned}
$$


Thus, by Proposition 3.5 with $Y_{1}=X_{\theta_{G} \wedge \delta}^{A}, Y_{2}=X, \Phi(s)=G(s, U(s)), \Psi(s)=$ $S(s)-\tilde{S}_{0}(s), \eta=\alpha, \theta=\zeta=\left(\delta-\theta_{G}\right)^{+}+\varepsilon / 2$ and $g(v)=\mathcal{M} D_{\delta}\left(A, A_{0}\right) v^{-1+\varepsilon / 2}$, we obtain

$$
\begin{aligned}
\| t & \mapsto \int_{0}^{t}\left[S(t-s)-\tilde{S}_{0}(t-s)\right] G(s, U(s)) \mathrm{d} W_{H}(s) \|_{V_{\mathrm{c}}^{\alpha, p}\left(\left[0, T_{0}\right] \times \Omega ; X\right)} \\
& \lesssim D_{\delta}\left(A, A_{0}\right) \sup _{0 \leq t \leq T_{0}}\left\|s \mapsto(t-s)^{-\alpha} G(s, U(s))\right\|_{L^{p}\left(\Omega ; \gamma\left(0, t ; H, X_{\theta_{G} \wedge \delta}^{A}\right)\right)} \\
& \leq D_{\delta}\left(A, A_{0}\right) M_{\gamma}(G)\|U\|_{V_{\mathrm{c}}^{\alpha, p}\left(\left[0, T_{0}\right] \times \Omega ; X\right)} \\
& \lesssim D_{\delta}\left(A, A_{0}\right) M_{\gamma}(G)\left(1+\left\|x_{0}\right\|_{L^{p}(\Omega ; X)}\right)
\end{aligned}
$$

where the penultimate line follows by estimate (32). Note that the implied constants are independent of $T_{0}$ and depend on $X_{0}$ only in terms of $\left\|P_{0}\right\|_{\mathcal{L}\left(X, X_{0}\right)}$ and on $A$ and $A_{0}$ only in terms of $\omega, \theta$, and $K$.

\section{Part $2 g$.}

Inserting (26)-(33) in (25), we obtain that there exists a constant $C>0$ independent of $x_{0}$ and $y_{0}$, depending on $X_{0}$ only in terms of $\left\|P_{0}\right\|_{\mathcal{L}\left(X, X_{0}\right)}$, on $A$ and $A_{0}$ only in terms of $1+D_{\delta}\left(A, A_{0}\right), \omega, \theta$, and $K$, and on $F$ and $G$ only in terms of their Lipschitz and linear growth constants $\operatorname{Lip}(F), \operatorname{Lip}_{\gamma}(G), M(F)$, and $M_{\gamma}(G)$, such that for all $T_{0} \in[0, T]$ one has

$$
\begin{aligned}
& \left\|U-\tilde{U}^{(0)}\right\|_{V_{\mathrm{c}}^{\alpha, p}\left(\left[0, T_{0}\right] \times \Omega ; X\right)} \leq C T_{0}^{\frac{1}{2} \varepsilon}\left\|U-\tilde{U}^{(0)}\right\|_{V_{\mathrm{c}}^{\alpha, p}\left(\left[0, T_{0}\right] \times \Omega ; X\right)} \\
& +C\left(\left\|x_{0}-y_{0}\right\|_{L^{p}(\Omega ; X)}+D_{\delta}\left(A, A_{0}\right)\left(1+\left\|x_{0}\right\|_{L^{p}\left(\Omega ; X_{\delta}^{A}\right)}\right)\right) .
\end{aligned}
$$

Setting $T_{0}=[2 C]^{-2 / \varepsilon}$ we obtain

$$
\begin{aligned}
& \left\|U-\tilde{U}^{(0)}\right\|_{V_{\mathrm{c}}^{\alpha, p}\left(\left[0, T_{0}\right] \times \Omega ; X\right)} \\
& \quad \leq 2 C\left(\left\|x_{0}-y_{0}\right\|_{L^{p}(\Omega ; X)}+D_{\delta}\left(A, A_{0}\right)\left(1+\left\|x_{0}\right\|_{L^{p}\left(\Omega ; X_{\delta}^{A}\right)}\right)\right) .
\end{aligned}
$$

\section{Part 3.}

Let $t_{0} \geq 0, z \in L^{p}\left(\Omega, \mathcal{F}_{t_{0}} ; X\right), T>0$ and $\alpha \in\left[0, \frac{1}{2}\right)$. By $U\left(z, t_{0}, \cdot\right)$, we denote the (unique) process in $V_{\mathrm{c}}^{\alpha, p}\left(\left[t_{0}, t_{0}+T\right] \times \Omega ; X\right)$ satisfying, for $s \in\left[t_{0}, t_{0}+T\right]$,

$$
\begin{aligned}
U\left(z, t_{0}, s\right)= & S\left(t-t_{0}\right) z+\int_{t_{0}}^{t} S\left(t-t_{0}-s\right) F\left(U\left(z, t_{0}, s\right)\right) d s \\
& +\int_{t_{0}}^{t} S\left(t-t_{0}-s\right) G\left(U\left(z, t_{0}, s\right)\right) \mathrm{d} W_{H}(s) \text { a.s. }
\end{aligned}
$$

The process $U^{(0)}\left(z, t_{0}, \cdot\right)$ is defined analogously. 
From the proof of (34), it follows that for any $x \in L^{p}\left(\Omega, \mathcal{F}_{t_{0}} ; X_{\delta}^{A}\right)$ and $y \in$ $L^{p}\left(\Omega, \mathcal{F}_{t_{0}} ; X\right)$ we have

$$
\begin{aligned}
& \left\|U\left(x, t_{0}, \cdot\right)-U^{(0)}\left(y, t_{0}, \cdot\right)\right\|_{V_{\mathrm{c}}^{\alpha, p}\left(\left[t_{0}, t_{0}+T_{0}\right] \times \Omega ; X\right)} \\
& \quad \leq 2 C\left(\|x-y\|_{L^{p}(\Omega ; X)}+D_{\delta}\left(A, A_{0}\right)\left[1+\|x\|_{L^{p}\left(\Omega ; X_{\delta}^{A}\right)}\right]\right),
\end{aligned}
$$

with $C$ as in (34). The remainder of the proof is entirely analogous to part 4 of the proof of [5, Theorem 3.1], see also [3, Theorem 6.2].

It remains to provide a proof for Proposition 4.4. For that purpose, we first prove the following lemma. Given the lemma, the proof of Proposition 4.4 basically follows the lines of known proofs concerning comparison of semigroups, see the monograph by ENGEL AND NAGEL [9, Chapter III.3.b]. For notational simplicity, we define the pseudo-resolvent

$$
R\left(\lambda: \tilde{A}_{0}\right):=i_{X_{0}} R\left(\lambda: A_{0}\right) P_{0}, \quad \lambda \in \omega+\Sigma_{\frac{\pi}{2}+\theta}
$$

(we leave it to the reader to verify the resolvent identity).

LEMMA 4.5. Let the setting be as in Proposition 4.4. Then for all $\lambda \in \omega^{\prime}+\sum_{\frac{\pi}{2}}+\theta$ we have

$$
\begin{aligned}
& \left\|R(\lambda: A)-R\left(\lambda: \tilde{A}_{0}\right)\right\|_{\mathcal{L}\left(X_{\beta}^{A}, X\right)} \\
& \quad \leq C_{\omega, \theta, K, P_{0}, \lambda_{0}}|\lambda-\omega|^{\delta-\beta-1}\left\|R\left(\lambda_{0}: A\right)-R\left(\lambda_{0}: \tilde{A}_{0}\right)\right\|_{\mathcal{L}\left(X_{\delta-1}^{A}, X\right)},
\end{aligned}
$$

where $C_{\omega, \theta, K, P_{0}, \lambda_{0}}$ is a constant depending only on $\lambda_{0}, \omega, \theta, K$ and $\left\|P_{0}\right\|_{\mathcal{L}\left(X, X_{0}\right)}$.

Proof. Using only the resolvent identity and the definition of $R\left(\lambda: \tilde{A}_{0}\right)$ (see (36)) one may verify that the following identity holds:

$$
\begin{aligned}
& R(\lambda: A)-R\left(\lambda: \tilde{A}_{0}\right) \\
& \quad=\left[I+\left(\lambda_{0}-\lambda\right) R\left(\lambda: \tilde{A}_{0}\right)\right]\left[R\left(\lambda_{0}: A\right)-R\left(\lambda_{0}: \tilde{A}_{0}\right)\right]\left(\lambda_{0}-A\right) R(\lambda: A) .
\end{aligned}
$$

Moreover, one may check that

$$
\omega^{\prime}+\Sigma_{\frac{\pi}{2}+\theta} \subset\left(\omega+\Sigma_{\frac{\pi}{2}+\theta}\right) \bigcap\left\{\lambda \in \mathbb{C}:|\lambda-\omega| \geq\left|\lambda_{0}-\omega\right|\right\} .
$$

Therefore one has, for $\lambda \in \omega^{\prime}+\Sigma_{\frac{\pi}{2}+\theta}$,

$$
\left\|I+\left(\lambda_{0}-\lambda\right) R\left(\lambda: \tilde{A}_{0}\right)\right\|_{\mathcal{L}(X)} \leq 1+\frac{\left|\lambda_{0}-\lambda\right|}{|\lambda-\omega|} K\left\|P_{0}\right\|_{\mathcal{L}\left(X, X_{0}\right)} \leq 1+2 K\left\|P_{0}\right\|_{\mathcal{L}\left(X, X_{0}\right)} .
$$

From (37), one obtains

$$
\begin{aligned}
& \left\|R(\lambda: A)-R\left(\lambda: \tilde{A}_{0}\right)\right\|_{\mathcal{L}\left(X_{\beta}^{A}, X\right)} \leq\left(1+2 K\left\|P_{0}\right\|_{\mathcal{L}\left(X, X_{0}\right)}\right) \\
& \quad \times\left\|R\left(\lambda_{0}: A\right)-R\left(\lambda_{0}: \tilde{A}_{0}\right)\right\|_{\mathcal{L}\left(X_{\delta-1}^{A}, X\right)}\left\|\left(\lambda_{0}-A\right) R(\lambda: A)\right\|_{\mathcal{L}\left(X_{\beta}^{A}, X_{\delta-1}^{A}\right)}
\end{aligned}
$$


Recall from page 811 that $X_{\beta}^{A}, \beta \in \mathbb{R}$, is defined in terms of $((\omega+1) I-A)^{\beta}$. Thus

$$
\begin{aligned}
& \left\|\left(\lambda_{0} I-A\right) R(\lambda: A)\right\|_{\mathcal{L}\left(X_{\beta}^{A}, X_{\delta-1}^{A}\right)}=\left\|((\omega+1) I-A)^{\delta-\beta-1}\left(\lambda_{0}-A\right) R(\lambda: A)\right\|_{\mathcal{L}(X)} \\
& \quad \leq\left(1+\left|\omega+1-\lambda_{0}\right| K\right)\left\|((\omega+1) I-A)^{\delta-\beta} R(\lambda: A)\right\|_{\mathcal{L}(X) .}
\end{aligned}
$$

By choice of $\lambda$, we have $|\lambda-\omega| \geq|| \lambda|-\omega| \geq|\omega+1-\omega|=1$, whence for $\delta-\beta=1$ we have

$$
\begin{aligned}
\left\|((\omega+1) I-A)^{\delta-\beta} R(\lambda: A)\right\|_{\mathcal{L}(X)} & =\|((\omega+1) I-A) R(\lambda: A)\|_{\mathcal{L}(X)} \\
& \leq 1+\left|\frac{\lambda-\omega-1}{\lambda-\omega}\right| K \leq 1+2 K .
\end{aligned}
$$

If $\delta-\beta=0$, then

$$
\left\|((\omega+1) I-A)^{\delta-\beta} R(\lambda: A)\right\|_{\mathcal{L}(X)}=\|R(\lambda: A)\|_{\mathcal{L}(X)} \leq K|\lambda-\omega|^{-1} .
$$

For $\delta-\beta \in(0,1)$ we have, by estimate (4),

$$
\begin{aligned}
& \left\|((\omega+1) I-A)^{\delta-\beta} R(\lambda: A)\right\|_{\mathcal{L}(X)} \\
& \quad \leq 2(1+K)\|R(\lambda: A)\|_{\mathcal{L}(X)}^{1+\beta-\delta}\|((\omega+1) I-A) R(\lambda: A)\|_{\mathcal{L}(X)}^{\delta-\beta} \\
& \quad \leq 2(1+K)(1+2 K)^{\delta-\beta} K^{1+\beta-\delta}|\lambda-\omega|^{\delta-\beta-1} \\
& \quad \leq 2(1+2 K)^{2}|\lambda-\omega|^{\delta-\beta-1} .
\end{aligned}
$$

Substituting this into (38) one obtains

$$
\begin{aligned}
& \left\|R(\lambda: A)-R\left(\lambda: \tilde{A}_{0}\right)\right\|_{\mathcal{L}\left(X_{\beta}^{A}, X\right)} \leq 2(1+2 K)^{3}\left(1+\left|\lambda_{0}-\omega-1\right| K\right)\left\|P_{0}\right\|_{\mathcal{L}\left(X, X_{0}\right)} \\
& \quad \times|\lambda-\omega|^{\delta-\beta-1}\left\|R\left(\lambda_{0}: A\right)-R\left(\lambda_{0}: \tilde{A}_{0}\right)\right\|_{\mathcal{L}\left(X_{\delta-1}^{A}, X\right)} .
\end{aligned}
$$

Proof of Proposition 4.4. Let $\omega^{\prime}$ be as defined in Lemma 4.5. For brevity set $\varepsilon=\delta-\beta$. Fix $\theta^{\prime} \in(0, \theta)$. It follows from [20, Theorem 1.7.7], that one has, for all $t>0$,

$$
S(t)=\frac{1}{2 \pi i} \int_{\omega^{\prime}+\Gamma_{\theta^{\prime}}} e^{\lambda t} R(\lambda: A) d \lambda ;
$$

where $\Gamma_{\theta^{\prime}}$ is the path composed from the two rays $r e^{i\left(\frac{\pi}{2}+\theta^{\prime}\right)}$ and $r e^{-i\left(\frac{\pi}{2}+\theta^{\prime}\right)}, 0 \leq r<$ $\infty$, and is oriented such that $\Im m(\lambda)$ increases along $\Gamma_{\theta^{\prime}}$. As $\omega^{\prime} \geq \omega$, the integral is well-defined as $\mathcal{L}(X)$-valued Bochner integral, and for $t>0$ one has

$$
S^{\prime}(t)=\frac{1}{2 \pi i} \int_{\omega^{\prime}+\Gamma_{\theta^{\prime}}} \lambda e^{\lambda t} R(\lambda: A) d \lambda ;
$$

the integral again being well-defined as $\mathcal{L}(X)$-valued Bochner integrals (see also the proof of [20, Theorem 2.5.2]). Analogous identities hold for $\tilde{S}_{0}$ and $R\left(\lambda: \tilde{A}_{0}\right)$.

First, let us assume that $\varepsilon \in(0,1)$. Below, we apply Lemma 4.5 , observing that for $r \in[0, \infty)$, we have 


$$
\left|\omega^{\prime}+r e^{ \pm i\left(\frac{\pi}{2}+\theta^{\prime}\right)}-\omega\right| \geq K_{\theta} r,
$$

where $K_{\theta}$ is a constant depending only on $\theta$. Note that we use the coordinate transform $\lambda=\omega^{\prime}+r e^{ \pm i\left(\frac{\pi}{2}+\theta^{\prime}\right)}$. For $s>0$, we have

$$
\begin{aligned}
& \left\|S(s)-\tilde{S}_{0}(s)\right\|_{\mathcal{L}\left(X_{\beta}^{A}, X\right)}=\left\|\frac{1}{2 \pi i} \int_{\omega^{\prime}+\Gamma_{\theta^{\prime}}} e^{\lambda s}\left[R(\lambda: A)-R\left(\lambda: \tilde{A}_{0}\right)\right] d \lambda\right\|_{\mathcal{L}\left(X_{\beta}^{A}, X\right)} \\
& \leq \frac{1}{2 \pi} \int_{0}^{\infty}\left|e^{\left(\omega^{\prime}+r e^{-i\left(\frac{\pi}{2}+\theta^{\prime}\right)}\right) s}\right| \\
& \quad \times\left\|R\left(\omega^{\prime}+r e^{-i\left(\frac{\pi}{2}+\theta^{\prime}\right)}: A\right)-R\left(\omega^{\prime}+r e^{-i\left(\frac{\pi}{2}+\theta^{\prime}\right)}: \tilde{A}_{0}\right)\right\|_{\mathcal{L}\left(X_{\beta}^{A}, X\right)} d r \\
& \quad+\frac{1}{2 \pi} \int_{0}^{\infty} \mid e^{\left(\omega^{\prime}+r e^{i\left(\frac{\pi}{2}+\theta^{\prime}\right)}\right) s \mid} \\
& \quad \times\left\|R\left(\omega^{\prime}+r e^{i\left(\frac{\pi}{2}+\theta^{\prime}\right)}: A\right)-R\left(\omega^{\prime}+r e^{i\left(\frac{\pi}{2}+\theta^{\prime}\right)}: \tilde{A}_{0}\right)\right\|_{\mathcal{L}\left(X_{\beta}^{A}, X\right)} d r \\
& \leq \\
& =\frac{1}{\pi} C_{\omega, \theta, K, P_{0}, \lambda_{0}} K_{\theta} D_{\delta}\left(A, A_{0}\right) e^{\omega^{\prime} s} \int_{0}^{\infty} r^{\varepsilon-1} e^{-r s \sin \theta^{\prime}} d r \\
& =\frac{\Gamma(\varepsilon)}{\pi}\left[\sin \theta^{\prime}\right]^{-\varepsilon} C_{\omega, \theta, K, P_{0}, \lambda_{0}} D_{\delta}\left(A, A_{0}\right) s^{-\varepsilon} e^{\omega^{\prime} s} .
\end{aligned}
$$

For $\varepsilon=0$, one may obtain the desired estimate by avoiding the singularity in 0 in the usual way: for $s>0$ given, we integrate over

$$
\omega^{\prime}+\Gamma_{\theta^{\prime}, s}=\left(\omega^{\prime}+\Gamma_{\theta^{\prime}, s}^{(1)}\right) \cup\left(\omega^{\prime}+\Gamma_{\theta^{\prime}, s}^{(2)}\right) \cup\left(\omega^{\prime}+\Gamma_{\theta^{\prime}, s}^{(3)}\right),
$$

where $\Gamma_{\theta^{\prime}, s}^{(1)}$ and $\Gamma_{\theta^{\prime}, s}^{(2)}$ are the rays $r e^{i\left(\frac{\pi}{2}+\theta^{\prime}\right)}$ and $r e^{-i\left(\frac{\pi}{2}+\theta^{\prime}\right)}, s^{-1} \leq r<\infty$, and $\Gamma_{\theta^{\prime}, s}^{(3)}=s^{-1} e^{i \phi}, \phi \in\left[-\frac{\pi}{2}-\theta^{\prime}, \frac{\pi}{2}+\theta^{\prime}\right]$ (we leave the details to the reader).

Recalling that $\varepsilon=\delta-\beta$, this proves the uniform boundedness estimate of (20).

In a similar fashion as above, for $\varepsilon \in[0,1]$ and $s>0$ we have

$$
\begin{aligned}
\left\|S^{\prime}(s)-\tilde{S}_{0}^{\prime}(s)\right\|_{\mathcal{L}\left(X_{\beta}^{A}, X\right)} & =\left\|\frac{1}{2 \pi i} \int_{\omega^{\prime}+\Gamma_{\theta^{\prime}}} \lambda e^{\lambda S}\left[R(\lambda: A)-R\left(\lambda: \tilde{A}_{0}\right)\right] d \lambda\right\|_{\mathcal{L}\left(X_{\beta}^{A}, X\right)} \\
& \leq \frac{1}{\pi} C_{\omega, \theta, K, P_{0}, \lambda_{0}} K_{\theta} D_{\delta}\left(A, A_{0}\right)\left[s \sin \theta^{\prime}\right]^{-1-\varepsilon} e^{\omega^{\prime} s} \int_{0}^{\infty} u^{\varepsilon} e^{-u} \mathrm{~d} u \\
& =\frac{\varepsilon \Gamma(\varepsilon)}{\pi}\left[\sin \theta^{\prime}\right]^{-1-\varepsilon} C_{\omega, \theta, K, P_{0}, \lambda_{0}} K_{\theta} D_{\delta}\left(A, A_{0}\right) s^{-1-\varepsilon} e^{\omega^{\prime} s} .
\end{aligned}
$$

Recalling that $\varepsilon=\delta-\beta$ this proves the uniform boundedness estimate of (21).

Concerning the $\gamma$-boundedness estimates, fix $\alpha>\varepsilon$. By Proposition 2.9, one has

$$
\begin{gathered}
\gamma_{\left[X_{\beta}^{A}, X\right]}\left(\left\{s^{\alpha}\left[S(s)-\tilde{S}_{0}(s)\right]: s \in[0, t]\right\}\right) \leq \int_{0}^{t}\left\|\left[s^{\alpha}\left(S(s)-\tilde{S}_{0}(s)\right)\right]^{\prime}\right\|_{\mathcal{L}\left(X_{\beta}^{A}, X\right)} d s \\
\leq \int_{0}^{t} \alpha s^{\alpha-1}\left\|S(s)-\tilde{S}_{0}(s)\right\|_{\mathcal{L}\left(X_{\beta}^{A}, X\right)} d s+\int_{0}^{t} s^{\alpha}\left\|S^{\prime}(s)-\tilde{S}_{0}^{\prime}(s)\right\|_{\mathcal{L}\left(X_{\beta}^{A}, X\right)} d s .
\end{gathered}
$$

Substituting (20) and (21) into the above and using that $\alpha>\varepsilon$ one obtains that there exists a constant $C$ depending only on $\lambda_{0}, \omega, \theta, K, \varepsilon=\delta-\beta, \alpha$, and $\left\|P_{0}\right\|_{\mathcal{L}\left(X, X_{0}\right)}$ such that 


$$
\begin{aligned}
\gamma_{\left[X_{\beta}^{A}, X\right]}\left(\left\{s^{\alpha}\left[S(s)-\tilde{S}_{0}(s)\right]: s \in[0, t]\right\}\right) & \leq C D_{\delta}\left(A, A_{0}\right) \int_{0}^{t} s^{\alpha-1-\varepsilon} e^{\omega^{\prime} s} e^{\bar{\omega} t} d s \\
& \leq C t^{\alpha-\varepsilon} e^{\left(\omega^{\prime} T\right)^{+}} D_{\delta}\left(A, A_{0}\right) .
\end{aligned}
$$

COROLLARY 4.6. Let the setting be as in Theorem 4.3. Let $\lambda \in\left(0, \frac{1}{2}\right)$ satisfy

$$
\lambda<\min \left\{1-\left(\delta-\theta_{F}\right)^{+}, \frac{1}{2}-\frac{1}{p}-\left(\delta-\theta_{G}\right)^{+}\right\} .
$$

Suppose $x_{0} \in L^{p}\left(\Omega, \mathcal{F}_{0} ; X_{\delta}^{A}\right)$ and $y_{0} \in L^{p}\left(\Omega, \mathcal{F}_{0} ; X\right)$, then

$$
\begin{aligned}
& \left\|U-S x_{0}-i_{X_{0}}\left(U^{(0)}-S_{0} P_{0} y_{0}\right)\right\|_{L^{p}\left(\Omega ; C^{\lambda}([0, T] ; X)\right)} \\
& \quad \lesssim\left\|x_{0}-y_{0}\right\|_{L^{p}(\Omega, X)}+D_{\delta}\left(A, A_{0}\right)\left(1+\left\|x_{0}\right\|_{L^{p}\left(\Omega ; X_{\delta}^{A}\right)}\right),
\end{aligned}
$$

with implied constant depending on $X_{0}$ only in terms of $\left\|P_{0}\right\|_{\mathcal{L}\left(X, X_{0}\right)}$, on $A$ and $A_{0}$ only in terms of $1 \vee D_{\delta}\left(A, A_{0}\right), \omega, \theta$ and $K$, and on $F$ and $G$ only in terms of their Lipschitz and linear growth constants $\operatorname{Lip}(F), \operatorname{Lip}_{\gamma}(G), M(F)$, and $M_{\gamma}(G)$.

Proof. As before, we write

$$
\begin{aligned}
\| U & -S x_{0}-i_{X_{0}}\left(U^{(0)}-S_{0} P_{0} y_{0}\right) \|_{L^{p}\left(\Omega ; C^{\lambda}([0, T] ; X)\right)} \\
=\| t & \mapsto \int_{0}^{t} \tilde{S}_{0}(t-s)\left[F(s, U(s))-F\left(s, \tilde{U}^{(0)}(s)\right)\right] d s \|_{L^{p}\left(\Omega ; C^{\lambda}([0, T] ; X)\right)} \\
& +\left\|t \mapsto \int_{0}^{t}\left[S(t-s)-\tilde{S}_{0}(t-s)\right] F(s, U(s)) d s\right\|_{L^{p}\left(\Omega ; C^{\lambda}([0, T] ; X)\right)} \\
& +\left\|t \mapsto \int_{0}^{t} \tilde{S}_{0}(t-s)\left[G(s, U(s))-G\left(s, \tilde{U}^{(0)}(s)\right)\right] \mathrm{d} W_{H}(s)\right\|_{L^{p}\left(\Omega ; C^{\lambda}([0, T] ; X)\right)} \\
& +\left\|t \mapsto \int_{0}^{t}\left[S(t-s)-\tilde{S}_{0}(t-s)\right] G(s, U(s)) \mathrm{d} W_{H}(s)\right\|_{L^{p}\left(\Omega ; C^{\lambda}([0, T] ; X)\right)} \cdot
\end{aligned}
$$

For the first and second term on the right-hand side of (39), we apply Proposition 3.6. Note that as before we may pick $\eta_{F}, \eta_{G} \leq 0$ such that $\eta_{F}<\theta_{F}-\delta$ and $\eta_{G}<\theta_{G}-\delta$ and

$$
\lambda<\min \left\{1+\eta_{F}, \frac{1}{2}-\frac{1}{p}+\eta_{G}\right\} .
$$

Our choice of $Y_{1}, Y_{2}, \Phi, \Psi$ is the same as in part 2c, respectively $2 \mathrm{~d}$, of the proof of Theorem 4.3, whereas we set $\eta=1-\lambda$. This leads to the following estimates:

$$
\begin{aligned}
\| t & \mapsto \int_{0}^{t} \tilde{S}_{0}(t-s)\left[F(s, U(s))-F\left(s, \tilde{U}^{(0)}(s)\right)\right] d s \|_{L^{p}\left(\Omega ; C^{\lambda}([0, T] ; X)\right)} \\
& \lesssim\left\|U-\tilde{U}^{(0)}\right\|_{L^{p}\left(\Omega ; L^{\infty}(0, T ; X)\right)} \leq\left\|U-\tilde{U}^{(0)}\right\|_{V_{\mathrm{c}}^{\alpha, p}([0, T] \times \Omega ; X)},
\end{aligned}
$$


and

$$
\begin{aligned}
\| t & \mapsto \int_{0}^{t}\left[S(t-s)-\tilde{S}_{0}(t-s)\right] F(s, U(s)) d s \|_{L^{p}\left(\Omega ; C^{\lambda}([0, T] ; X)\right)} \\
& \lesssim D_{\delta}\left(A, A_{0}\right)\|U\|_{L^{p}\left(\Omega ; L^{\infty}(0, T ; X)\right)} \lesssim D_{\delta}\left(A, A_{0}\right)\left(1+\left\|x_{0}\right\|_{L^{p}(\Omega, X)}\right) .
\end{aligned}
$$

For the third and fourth term on the right-hand side of (39), we apply Corollary 3.4 with $\theta=\lambda$ and $\alpha \in\left(0, \frac{1}{2}\right)$ such that $\alpha>\lambda+\frac{1}{p}+\eta_{G}$. The choice of $Y_{1}, Y_{2}, \Phi$, and $\Psi$ is as in parts 2 e and 2 f of the proof of Theorem 4.3. This leads to the following:

$$
\begin{aligned}
\| t & \mapsto \int_{0}^{t} \tilde{S}_{0}(t-s)\left[G(s, U(s))-G\left(s, \tilde{U}^{(0)}(s)\right)\right] \mathrm{d} W_{H}(s) \|_{L^{p}\left(\Omega ; C^{\lambda}([0, T] ; X)\right)} \\
& \lesssim\left\|U-\tilde{U}^{(0)}\right\|_{V_{\mathrm{c}}^{\alpha, p}([0, T] \times \Omega ; X)},
\end{aligned}
$$

and

$$
\begin{aligned}
\| t & \mapsto \int_{0}^{t}\left[S(t-s)-\tilde{S}_{0}(t-s)\right] G(s, U(s)) \mathrm{d} W_{H}(s) \|_{L^{p}\left(\Omega ; C^{\lambda}([0, T] ; X)\right)} \\
& \lesssim D_{\delta}\left(A, A_{0}\right)\|U\|_{V_{\mathrm{c}}^{\alpha, p}([0, T] \times \Omega ; X)} \lesssim D_{\delta}\left(A, A_{0}\right)\left(1+\left\|x_{0}\right\|_{L^{p}(\Omega, X)}\right) .
\end{aligned}
$$

Combining these estimates with Theorem 4.3 gives the desired result. It goes without saying that all the implied constants above depend on $X_{0}$ only in terms of $\left\|P_{0}\right\|_{\mathcal{L}\left(X, X_{0}\right)}$, on $A$ and $A_{0}$ only in terms of $1+D_{\delta}\left(A, A_{0}\right), \omega, \theta$, and $K$, and on $F$ and $G$ only in terms of their Lipschitz and linear growth constants $\operatorname{Lip}(F), \operatorname{Lip}_{\gamma}(G), M(F)$, and $M_{\gamma}(G)$.

\section{Yosida approximations}

Consider equation (SDE) on a Banach space $X$ under the assumptions $(\mathbf{X}),(\mathbf{A}),(\mathbf{F})$, and $(\mathbf{G})$ with the additional assumption that $\theta_{F}, \theta_{G} \geq 0$. We can also assume, without loss of generality, that $A$ is of type $(\omega, K, \theta)$ for some $\omega \geq 0, \theta \in\left(0, \frac{1}{2}\right]$ and $K>0$. We define $A_{n}:=n A R(n: A)$ to be the $n^{\text {th }}$ Yosida approximation of $A$. Let $U$ denote the solution to equation (SDE) with operator $A$ and initial data $x_{0}$ and, for $n \in \mathbb{N}$, let $U^{(n)}$ denote the solution to equation (SDE) with operator $A_{n}$ instead of $A$ and initial data $y_{0} \in L^{p}\left(\Omega, \mathcal{F}_{0} ; X\right)$.

THEOREM 5.1. For any $\eta \geq 0$ and $p \in(2, \infty)$ such that

$$
\eta<\min \left\{\frac{3}{2}-\frac{1}{\tau}+\theta_{F}, \frac{1}{2}-\frac{1}{p}+\theta_{G}\right\}
$$

and any $\alpha \in\left[0, \frac{1}{2}\right)$ we have, assuming $x_{0} \in L^{p}\left(\Omega, \mathcal{F}_{0} ; X\right)$ and $y_{0} \in L^{p}\left(\Omega, \mathcal{F}_{0} ; X_{\eta}^{A}\right)$,

$$
\left\|U-U^{(n)}\right\|_{V_{\mathrm{c}}^{\alpha, p}([0, T] \times \Omega ; X)} \lesssim\left\|x_{0}-y_{0}\right\|_{L^{p}(\Omega ; X)}+n^{-\min \{\eta, 1\}}\left(1+\left\|y_{0}\right\|_{L^{p}\left(\Omega ; X_{\eta}^{A}\right)}\right),
$$

with implied constants independent of $n, x_{0}$ and $y_{0}$. 
The following corollary is a direct consequence of the Borel-Cantelli lemma and the above theorem (see e.g., [13, Lemma 2.1]):

COROLLARY 5.2. Let $\eta>0$ and $p \in(2, \infty)$ be such that

$$
\eta+\frac{1}{p}<\min \left\{\frac{3}{2}-\frac{1}{\tau}+\theta_{F}, \frac{1}{2}-\frac{1}{p}+\theta_{G}, 1\right\}
$$

and assume $y_{0}=x_{0} \in L^{p}\left(\Omega, \mathcal{F}_{0} ; X_{\eta}^{A}\right)$. Then there exists a random variable $\chi \in$ $L^{p}(\Omega)$ such that for all $n \in \mathbb{N}$

$$
\left\|U-U^{(n)}\right\|_{C([0, T] ; X)} \leq \chi n^{-\eta} .
$$

To prove Theorem 5.1 we need the following lemma:

LEMMA 5.3. Let $\beta \in[0,1]$. Then there exists a constant $K^{\prime}$ such that for all $n \geq 2 \omega$ and all $x \in X_{\beta}^{A}$ one has

$$
\left\|\left(2 \omega I-A_{n}\right)^{\beta} x\right\|_{\mathcal{L}(X)} \leq K^{\prime}\left\|(2 \omega I-A)^{\beta} x\right\|_{X} .
$$

Proof. Observe that

$$
\begin{aligned}
2 \omega I-A_{n} & =[2 \omega n I-(n+2 \omega) A] R(n: A) \\
& =\left[(n+2 \omega) I-4 \omega^{2} R(2 \omega: A)\right](2 \omega I-A) R(n: A) .
\end{aligned}
$$

Thus for $x \in D(A)$ and $n \geq 2 \omega$, we have

$$
\begin{aligned}
\left\|\left(2 \omega I-A_{n}\right) x\right\|_{X} & \leq\left\|\left[(n+2 \omega) I-4 \omega^{2} R(2 \omega: A)\right] R(n: A)\right\|_{\mathcal{L}(X)\|(2 \omega I-A) x\|_{X}} \\
& \leq\left[K \frac{n+2 \omega}{n-\omega}+K^{2} \frac{4 \omega}{n-\omega}\right]\|(2 \omega I-A) x\|_{X} \\
& \leq 4 K(1+K)\|(2 \omega I-A) x\|_{X} .
\end{aligned}
$$

This proves the lemma for $\beta=1$. For $\beta=0$, the lemma is trivial. For $\beta \in(0,1)$, we need two extra observations.

First of all, for $s>\omega$ and $\beta \in(0,1)$ we have, by definition (see [20, Section 2.6]):

$$
\begin{aligned}
\left\|(s I-A)^{-\beta} x\right\|_{X} & =\left\|\frac{\sin (\pi \beta)}{\pi} \int_{0}^{\infty} t^{-\beta}((t+s) I-A)^{-1} x \mathrm{~d} t\right\|_{X} \\
& \leq K \frac{\sin (\pi \beta)}{\pi} \int_{0}^{\infty} t^{-\beta}(t+s-\omega)^{-1} \mathrm{~d} t\|x\|_{X} \\
& \leq K \frac{\sin (\pi \beta)}{\pi \beta(1-\beta)}(s-\omega)^{-\beta}\|x\|_{X} .
\end{aligned}
$$

Second, let $\mu, \lambda \in \omega+\Sigma_{\frac{\pi}{2}+\theta}$. We have the following:

$$
\left\|e^{-(\lambda I-A) R(\mu: A) t}\right\|_{\mathcal{L}(X)}=e^{-t}\left\|e^{(\mu-\lambda) R(\mu, A) t}\right\|_{\mathcal{L}(X)} \leq e^{-t+\frac{|\mu-\lambda|}{|\mu-\omega|} K t} .
$$

Now, suppose $n \geq 2 \omega(1+4 K), \lambda=2 \omega, \mu=\frac{n \lambda}{\lambda+n}=\frac{2 \omega n}{2 \omega+n}$. In that case, one may check that $\frac{|\mu-\lambda|}{|\mu-\omega|} K \leq \frac{1}{2}$, and thus that for $\beta \in(0,1)$, we have

$$
\begin{aligned}
& \left\|\left[-(2 \omega I-A) R\left(\frac{2 \omega n}{2 \omega+n}: A\right)\right]^{-\beta}\right\|_{\mathcal{L}(X)} \\
& =\left\|\frac{1}{\Gamma(\beta)} \int_{0}^{\infty} t^{\beta-1} e^{-(2 \omega I-A) R\left(\frac{2 \omega n}{2 \omega+n}: A\right) t} \mathrm{~d} t\right\|_{\mathcal{L}(X)} \leq 2^{\beta} .
\end{aligned}
$$


It follows that there exists a constant $M \geq 2^{\beta}$ such that for all $n \geq 2 \omega$ we have

$$
\left\|\left[-(2 \omega I-A) R\left(\frac{2 \omega n}{2 \omega+n}: A\right)\right]^{-\beta}\right\|_{\mathcal{L}(X)} \leq M .
$$

For $\beta \in(0,1)$ and $x \in X_{\beta}^{A}$ we have, by standard theory on functional calculus (see e.g., the monograph by HAASE [10]), equation (40), and the estimates (41) and (42), that

$$
\begin{aligned}
\left\|\left(2 \omega I-A_{n}\right)^{\beta} x\right\|_{X}= & \left\|(n+2 \omega)^{\beta}\left(\frac{2 \omega n}{2 \omega+n} I-A\right)^{\beta}(n I-A)^{-\beta} x\right\|_{X} \\
\leq & (n+2 \omega)^{\beta}\left\|\left[-(2 \omega I-A) R\left(\frac{2 \omega n}{2 \omega+n}: A\right)\right]^{-\beta}\right\|_{\mathcal{L}(X)} \\
& \times\left\|(2 \omega I-A)^{\beta} x\right\|_{X}\left\|(n I-A)^{-\beta}\right\|_{\mathcal{L}(X)} \\
\leq & 4^{\beta} \frac{\sin (\pi \beta)}{\pi \beta(1-\beta)} K M\left\|(2 \omega I-A)^{\beta} x\right\|_{X} .
\end{aligned}
$$

Proof of Theorem 5.1. In order to apply Theorem 4.3, we must prove that $A_{n}, n \geq 2 \omega$, are of uniform type, i.e., that there exist $\bar{\omega} \in \mathbb{R}, \bar{\theta} \in\left(0, \frac{\pi}{2}\right]$ and $\bar{K}>0$ such that $A_{n}$ is of type $(\bar{\omega}, \bar{\theta}, \bar{K})$ for all $n \geq 2 \omega$. Fix $n \geq 2 \omega$. One checks that

$$
R\left(\lambda: A_{n}\right)=(n+\lambda)^{-1}(n-A) R\left(\frac{\lambda n}{n+\lambda}: A\right)
$$

whenever $\frac{\lambda n}{n+\lambda} \in \omega+\Sigma_{\frac{\pi}{2}+\theta}$. By standard theory on Möbius transforms, it follows that $\varrho\left(A_{n}\right) \subset 2 \omega+\Sigma_{\frac{\pi}{2}+\theta}$ for $n \geq 2 \omega$.

Using (43) one may check that for $\lambda \in 2 \omega+\Sigma_{\frac{\pi}{2}+\theta}$ we have the following:

$$
R(\lambda: A)-R\left(\lambda: A_{n}\right)=-(\lambda+n)^{-1} A^{2} R\left(\frac{\lambda n}{n+\lambda}: A\right) R(\lambda: A) .
$$

Thus by (2) we have, for $\lambda \in \omega\left(1+2(\cos \theta)^{-1}\right)+\Sigma_{\frac{\pi}{2}+\theta}$,

$$
\left\|R(\lambda: A)-R\left(\lambda: A_{n}\right)\right\|_{\mathcal{L}(X)} \leq(1+2 K)^{2}|\lambda+n|^{-1} \leq(1+2 K)^{2}|\lambda-\omega|^{-1} .
$$

The final estimate follows from the fact that by standard theory on Möbius transforms we have that $\frac{|\lambda-\omega|}{|\lambda+n|} \leq 1$ for $\lambda \in \omega+\Sigma_{\frac{\pi}{2}+\theta}$ and $n \geq 2 \omega$. In conclusion, we have, for $\lambda \in \omega\left(1+2(\cos \theta)^{-1}\right)+\Sigma_{\frac{\pi}{2}+\theta}$,

$$
\begin{aligned}
\left\|R\left(\lambda: A_{n}\right)\right\|_{\mathcal{L}(X)} & \leq\|R(\lambda: A)\|_{\mathcal{L}(X)}+\left\|R(\lambda: A)-R\left(\lambda: A_{n}\right)\right\|_{\mathcal{L}(X)} \\
& \leq\left[K+(1+2 K)^{2}\right]|\lambda-\omega|^{-1}
\end{aligned}
$$

This proves that $A_{n}$ is of type $\left(\omega\left(1+2(\cos \theta)^{-1}\right), \theta, K+(1+2 K)^{2}\right)$ for all $n \geq 2 \omega$.

It also follows from (44) that if we take, for example, $\lambda_{0}=\omega\left(1+2(\cos \theta)^{-1}\right)$, then we have, for $n \geq 2 \omega$,

$$
\left\|R\left(\lambda_{0}: A\right)-R\left(\lambda_{0}: A_{n}\right)\right\|_{\mathcal{L}(X)} \leq(1+2 K)^{2} n^{-1} .
$$

In other words, for all $n \in \mathbb{N}$ condition (17) in Theorem 4.3 is satisfied with $X=X_{0}$, $\delta=1$ and $\lambda_{0}=\omega\left(1+2(\cos \theta)^{-1}\right)$. In particular, we can apply Theorem 4.3 to obtain 
the desired result for the case $\theta_{F}>-\frac{1}{2}+\frac{1}{\tau}$, where $\tau$ is the type of $X$, and $\theta_{G}>\frac{1}{2}+\frac{1}{p}$. Concerning the dependence on $1+D_{1}\left(A, A_{n}\right)$ of the implied constant in (19), note that $1+D_{1}\left(A, A_{n}\right)$ is uniformly bounded in $n$.

In order to get the desired result for general $\theta_{F}, \theta_{G} \geq 0$, we consider the difference $R\left(\lambda_{0}: A\right)-R\left(\lambda_{0}: A_{n}\right)$ in the $\mathcal{L}\left(X_{\delta-1}^{A_{n}}, X\right)$-norm. (Note that if $A$ is unbounded then $R\left(\lambda_{0}: A\right)-R\left(\lambda_{0}: A_{n}\right) \notin \mathcal{L}\left(X_{\delta-1}^{A}, X\right)$ for any $\delta<1$.) For $n \geq \omega\left(1+2(\cos \theta)^{-1}\right)$, we have, by (2), that $\left\|\left(2 \omega I-A_{n}\right)\right\|_{\mathcal{L}(X)} \leq 2 n(1+K)$. Thus by estimate (4), we have, for $\delta \in(0,1)$,

$$
\begin{aligned}
\left\|\left(2 \omega I-A_{n}\right)^{1-\delta} x\right\| & \leq 2(1+2 K)\|x\|^{\delta}\left\|A_{n} x\right\|_{X}^{1-\delta} \\
& \leq 2^{2-\delta}(1+2 K)^{2-\delta} n^{1-\delta}\|x\|_{X} .
\end{aligned}
$$

It follows that for $\delta \in[0,1)$ we have

$$
\left\|R\left(\lambda_{0}: A\right)-R\left(\lambda_{0}: A_{n}\right)\right\|_{\mathcal{L}\left(X_{\delta-1}^{A_{n}}, X\right)} \leq 2^{2-\delta}(1+2 K)^{4-\delta} n^{-\delta} .
$$

Observe that by Lemma 5.3 we have that $F:[0, T] \times X \rightarrow X_{\theta_{F}}^{A_{n}}$ is Lipschitz continuous and of linear growth for all $n \geq 2 \omega$ with Lipschitz and growth constants independent of $n$, and $G:[0, T] \times X \rightarrow \gamma\left(H, X_{\theta_{G}}^{A_{n}}\right)$ is $L_{\gamma}^{2}$-Lipschitz continuous and of linear growth for all $n \geq 2 \omega$ with Lipschitz and growth constants independent of $n$. Also, $1+D_{\delta}\left(A, A_{n}\right)$ is uniformly bounded in $n$.

Fix $\eta \in[0,1]$ such that $\eta<\min \left\{\frac{3}{2}-\frac{1}{\tau}+\theta_{F}, \frac{1}{2}-\frac{1}{p}+\theta_{G}\right\}$ and suppose $y_{0} \in$ $L^{p}\left(\Omega, \mathcal{F}_{0} ; X_{\eta}^{A}\right)$. It follows from Theorem 4.3 with $\delta=\eta$, but with $A_{n}$ playing the role of $A$ and $A$ playing the role of $A_{0}$ that

$$
\left\|U-U^{(n)}\right\|_{V_{\mathrm{c}}^{\alpha, p}([0, T] \times \Omega ; X)} \lesssim\left\|x_{0}-y_{0}\right\|_{L^{p}(\Omega ; X)}+n^{-\eta}\left(1+\left\|y_{0}\right\|_{L^{p}\left(\Omega ; X_{\eta}^{A}\right)}\right),
$$

with implied constants independent of $n, x_{0}$, and $y_{0}$.

\section{Acknowledgments}

The authors gratefully acknowledge Jan van Neerven and the anonymous referees for their helpful comments.

\section{REFERENCES}

[1] Z. Brzeźniak. On stochastic convolution in Banach spaces and applications. Stochastics Stochastics Rep., 61(3-4):245-295, 1997.

[2] D.L. Burkholder. Martingales and singular integrals in Banach spaces. In "Handbook of the Geometry of Banach Spaces”, Vol. I, pages 233-269. North-Holland, Amsterdam, 2001.

[3] S.G. Cox. Stochastic Differential Equations in Banach Spaces: Decoupling, Delay equations, and Approximations in Space and Time, 2012. PhD thesis, available online at http://repository.tudelft. nl.

[4] S.G. Cox and E. Hausenblas. Pathwise space approximations of semi-linear parabolic SPDEs with multiplicative noise. Int. J. Comput. Math., 89:2460-2478, 2012. 
[5] S.G. Cox and J.M.A.M. van Neerven. Pathwise Hölder convergence of the implicit-linear Euler scheme for semi-linear SPDEs with multiplicative noise. Numer. Math., 125(2):259-345, 2013.

[6] G. Da Prato, S. Kwapień, and J. Zabczyk. Regularity of solutions of linear stochastic equations in Hilbert spaces. Stochastics, 23(1):1-23, 1987.

[7] G. Da Prato and J. Zabczyk. "Stochastic Equations in Infinite Dimensions", volume 44 of Encyclopedia of Mathematics and its Applications. Cambridge University Press, Cambridge, 1992.

[8] W. Desch and W. Schappacher. A note on the comparison of $C_{0}$-semigroups. Semigroup Forum, 35(2):237-243, 1987.

[9] K.-J. Engel and R. Nagel. One-parameter semigroups for linear evolution equations, volume 194 of Graduate Texts in Mathematics. Springer-Verlag, New York, 2000.

[10] M.H.A. Haase. "The Functional Calculus for Sectorial Operators", volume 169 of Operator Theory: Advances and Applications. Birkhäuser Verlag, Basel, 2006.

[11] M. Jung. On the relationship between perturbed semigroups and their generators. Semigroup Forum, 61(2):283-297, 2000.

[12] N.J. Kalton and L. Weis. The $H^{\infty}$-calculus and square function estimates. In preparation.

[13] P.E. Kloeden and A. Neuenkirch. The pathwise convergence of approximation schemes for stochastic differential equations. LMS Journal of Comp. and Math., 10:235-253, 2007.

[14] M.C. Kunze and J.M.A.M. van Neerven. Approximating the coefficients in semilinear stochastic partial differential equations. J. Evol. Equ., 2011.

[15] M. Ledoux and M. Talagrand. Probability in Banach spaces, volume 23 of Ergebnisse der Mathematik und ihrer Grenzgebiete (3). Springer-Verlag, Berlin, 1991.

[16] J.M.A.M. van Neerven. $\gamma$-Radonifying operators - a survey. Proceedings of the CMA 44, pages $1-62,2010$.

[17] J.M.A.M. van Neerven and M.C. Veraar. On the stochastic Fubini theorem in infinite dimensions. In Stochastic partial differential equations and applications_-VII, volume 245 of Lect. Notes Pure Appl. Math., pages 323-336. Chapman \& Hall/CRC, Boca Raton, FL, 2006.

[18] J.M.A.M. van Neerven, M.C. Veraar, and L. Weis. Stochastic integration in UMD Banach spaces. Annals Probab., 35:1438-1478, 2007.

[19] J.M.A.M. van Neerven, M.C. Veraar, and L. Weis. Stochastic evolution equations in UMD Banach spaces. J. Funct. Anal., 255(4):940-993, 2008.

[20] A. Pazy. "Semigroups of Linear Operators and Applications to Partial Differential Equations", volume 44 of Applied Mathematical Sciences. Springer-Verlag, New York, 1983.

[21] D. Revuz and M. Yor. "Continuous Martingales and Brownian Motion", volume 293 of Grundlehren der Math. Wissenschaften. Springer-Verlag, Berlin, 3rd edition, 1999.

[22] D.W. Robinson. The approximation of flows. J. Functional Analysis, 24(3):280-290, 1977.

[23] R. Schnaubelt and M. Veraar. Structurally damped plate and wave equations with random point force in arbitrary space dimensions. Differential Integral Equations, 23(9-10):957-988, 2010.

[24] L. Weis. Operator-valued Fourier multiplier theorems and maximal $L_{p}$-regularity. Math. Ann., 319(4):735-758, 2001.

S. G. Cox

Seminar for Applied Mathematics,

ETH Zürich,

Zurich,

Switzerland

E-mail:sonja.cox@sam.math.ethz.ch

E. Hausenblas

Department of Mathematics and

Information Technology,

Montana Universität Leoben,

Leoben,

Austria

E-mail:erika.hausenblas@unileoben.ac.at 